\title{
In-situ calibration of galvanometric scanning head for laser powder bed fusion machines based on a vision system
}

Van Thao Le $e^{1,2}$ and Yann Quinsat ${ }^{3, *}$

${ }^{1}$ Institute of Research and Development, Duy Tan University, Da Nang 550000, Viet Nam.

${ }^{2}$ Advanced Technology Center, Le Quy Don Technical University, Hanoi, Vietnam.

${ }^{3}$ LURPA, ENS Paris-Saclay, Université Paris-Saclay, France.

*Corresponding author. Email: yann.quinsat@ens-paris-saclay.fr

\begin{abstract}
In Laser Powder Bed Fusion (LPBF) additive manufacturing machines, a laser galvanometric scanning system is used to control the movement of the laser beam, which melts material powders layer-by-layer in a powder bed. Errors between desirable positions and actual positions of the laser in the work plane of the machine directly affect the geometry accuracy of built parts. Therefore, calibrating the laser galvanometric scanning system of LPBF machines is a very important task. This system is generally calibrated by calculating correction tables for its joint components. However, most existing calibration methods require a measuring phase of actual positions of laser engraved on a calibration plate. This phase was generally performed by a measuring system in another location, thus requiring a delay time and causing an interruption of the calibration process. In the current study, an in-situ calibration method for the laser galvanometric scanning head of LPBF machines was developed based on a vision system. The vision system (i.e., a high-resolution monochrome camera) was used to acquire experimental data rapidly during the calibration process, thereby avoiding the laser marking and the measuring phases of laser marks in another location. The acquired experimental data was subsequently used to calculate the correction tables/matrices for calibrating the optical chain of an LPBF machine prototype. The obtained results show that the developed measurement method is acceptable for the in-situ measurement with a good accuracy. After the calibration process, errors between the positions generated by the identified LPBF machine and those measured by the proposed method are very small with an average error of $0.03 \mathrm{~mm}$, and about $80 \%$ of positions present an error less than $0.04 \mathrm{~mm}$ in the calibration region.
\end{abstract}

Keywords: Additive manufacturing, Laser powder bed fusion, Galvanometric scanning system, Insitu calibration, Vision system, Image processing. 


\section{Introduction}

\subsection{Context}

Laser galvanometric scanning systems are widely used in many fields such as medical imaging, optical metrology, and material processing due to its capability to achieve very high processing velocities, high-positioning repeatability, and small laser spot size [1-3]. In Laser Powder Bed Fusion (LPBF) additive manufacturing machines such as Selective Laser Melting (SLM) and Selective Laser Sintering (SLS), a laser galvanometric scanning head (Fig. 1) is also used to control the positions of laser spot in the work plane. This scanning head consists of a positioning device (i.e. two rotatable mirrors driven by two galvanometers) and a focusing device. The focusing device can be either an f-theta lens located after the positioning device, or a dynamic focus module (DFM) located before the positioning device [4-6]. The focusing device controls the laser spot size in the work plane, whereas the positioning device controls the positions of laser spot in the work plane. Errors between desired positions and actual positions of laser spot in the work plane directly affect the geometry accuracy of built parts. Therefore, the laser galvanometric scanning head of LPBF machines must be calibrated in an accurate manner to control lasing paths.

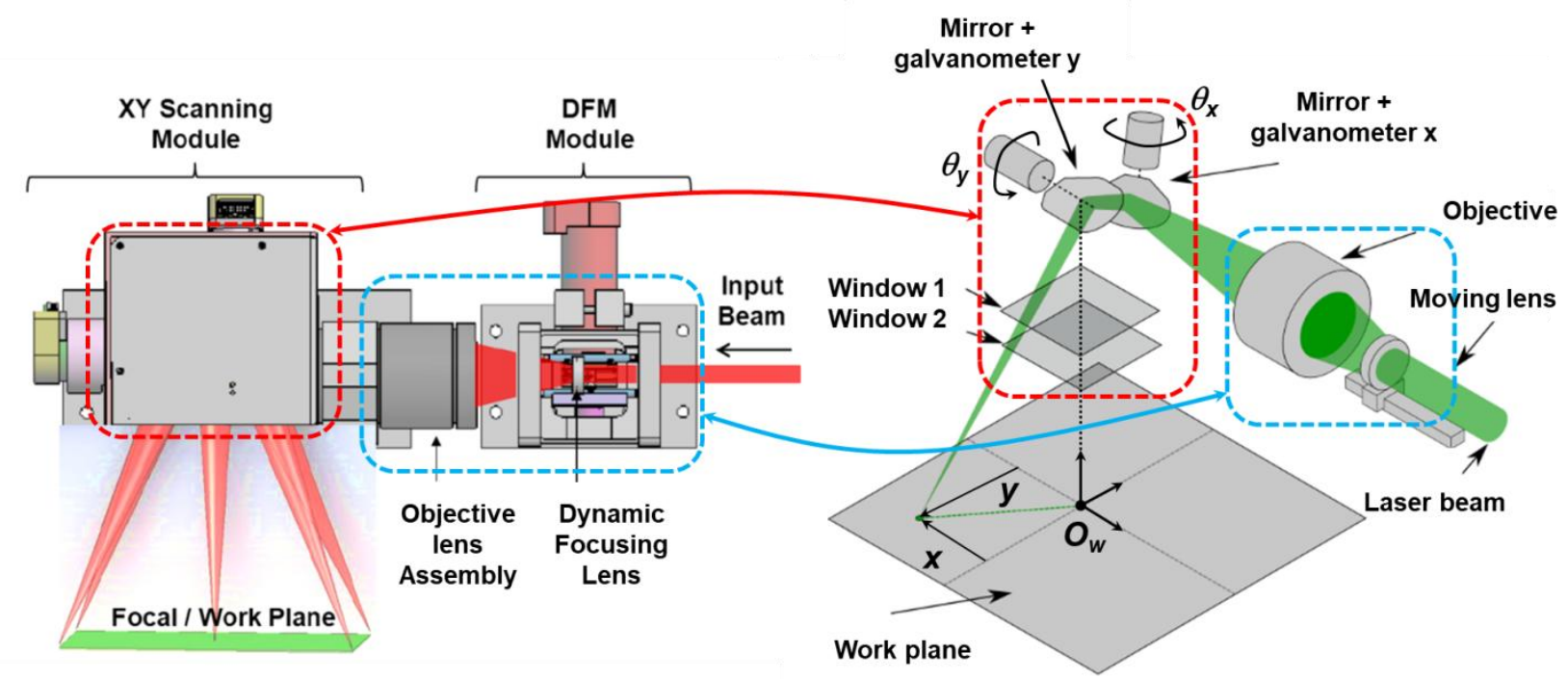

Fig. 1. Laser galvanometric scanning head of LPBF machines with dynamic focus module (DFM), adapted from [7].

\subsection{Literature review}

In the literature, much research has reported the calibration of galvanometric scanning systems. For instance, Mao et al. [8] and Yang et al. [9] reported the calibration of one-mirror galvanometric scanners, which were used in 3D measurement systems. Lüdtke et al. [10] and Tu et al. [11] calibrated two-mirror galvanometric laser scanners by using employed statistical learning methods (e.g., linear regression and artificial neural networks). For two-mirror galvanometric 
scanning systems, they were generally calibrated by calculating correction tables for all joint components - i.e., two rotatable mirrors. The correction tables allow us to determine the relationship between positions of laser spot in the work plane (also called the task space) and corresponding positions of all joint components in the join space.

Several mathematical models have been developed to describe the relationship between the task space and the joint space. For example, the models presented in $[12,13]$ have taken into account the geometry of all components of the laser galvanometric scanning system. However, these works have used many assumptions related to the geometry, for example, rotatable axes of mirrors confused with their reflection surfaces, and so on. Thereby, the real laser galvanometric scanning system was not modeled completely. To fill this gap, certain authors have developed more representative models by taking into account different sources of defects such as defects related to laser source orientation, positions and orientations of mirrors [14,15]. Recently, Godineau et al. [7] developed a complete analytical model for the laser galvanometric scanning head of LBPF machines. In their model, thirty defects related to all components of the laser scanning head were taken into consideration. Thus, the position of a laser spot in the work plane was determined as a function of all joint commands and thirty assembly defects.

In most of the published works [7,14-17], the calibration of laser galvanometric scanning systems by calculating correction tables was performed as follows: The laser beam was controlled to desired positions, which were defined by a grid of points. The laser beam engraved a grid of marks on a calibration plate to establish real positions of laser spot. Subsequently, the real positions of laser spot were measured by a measurement system in another location. Finally, based on mathematical models (with assembly defects [7] or without defects $[16,17]$ ) and experimental data, the correction tables/matrices were calculated. These methods allow the laser galvanometric scanning heads to be calibrated effectively and precisely. However, they were "rigid" and time consuming, because the steps of measuring the actual laser positions, which were engraved on the calibration plate was performed by a measuring system in another location. In addition, it is difficult to integrate such a measurement system in a closed-loop calibration procedure. Lastly, the measured data accuracy was significantly affected by the repositioning of the calibration plate and the deviations of the measuring systems.

Nowadays, vision inspection systems - also called machine vision systems are widely used for automated inspection, robot guidance, quality control, and manufacturing applications because of their accuracy, flexibility, repeatability and efficiency [18-21]. The vision inspection systems equipped with a camera or multiple cameras, and even video and lighting are capable of measuring parts, verifying parts in the correct position, and recognizing the shape of parts at high speeds. In 
the literature, many researchers have used such vision systems for the in-situ inspection/monitoring of AM processes [21-24]. In the vision inspection systems using a camera, the camera can be installed in a coaxial configuration or an off-axial configuration [23]. In the coaxial configuration, the camera follows the same optical path with the laser beam of LPBF systems to locally acquire melting pool signals. Therefore, the melting pool can be observed from the top view. This configuration was widely used for measuring the size and temperature of melting pool. On the other hand, in the off-axial configuration, the optical axis of the camera is not perpendicular to the work plane of LPBF machines. Thus, images captured by the camera always present perspective distortion. However, the images provide more comprehensive information, for example, melting pool shape in side views, temperature distribution, geometry and topography of deposited layers [25-30].

In the context of using a vision system for the calibration of a laser galvanometric scanner, Delgado and Lasagni [31] proposed a method using a self-designed calibration plate and a coaxial coupled vision device (i.e., a camera and an illumination source) that shared the same optical path with the laser beam. The authors demonstrated that their calibration method was capable of reducing the field distortion of the laser galvanometric scanner efficiently, and avoiding the necessity of repetitive marking and measuring phases of laser marks. However, this calibration method was only relied on experimental measurements. The geometry of the laser galvanometric scanning system was not taken into account in the calibration process.

\subsection{Research aim}

In this study, taking into account the complete analytical model of the laser galvanometric scanning head developed in [7] and the advantages of a vision system simultaneously, an in-situ calibration method for the laser galvanometric scanning head of LBPF machines was proposed based on a vision system with a high-resolution monochrome camera. The aim is to acquire experimental data rapidly during the calibration process, and avoid the laser marking and the measuring phases of laser marks. The research work presented in this paper was carried out in two phases: Firstly, the feasibility of using such a vision system to measure 2D objects in a plane with four available reference points was demonstrated. Secondly, the proposed measurement method was used to acquire the positions of laser spot intended for the calibration of the optical chain of LPBF machines according to the algorithm developed in [7].

This paper is organized as follows: an overview of the proposed method is described in Section 2. Section 3 details the materials and experimental setup. In Section 4, the intrinsic parameters of the camera are estimated via the camera calibration process. These parameters are used for the image processing and the measurement. The homography computation for correcting 
perspective distortion of images is also presented. In Section 5, the method for acquiring laser spot positions in the work plane of the LPBF machine is presented. The measured positions of laser spots are subsequently used for the calculation of correction tables to calibrate the optical chain of the LPBF machines (Section 6). Section 7 is intended for some conclusions and future works.

\section{Method overview}

An in-situ calibration method for the optical chain of LPBF machines was proposed in this study basing on a vision system - i.e., a high-resolution monochrome camera. In this method, the measurement of laser spot positions was directly performed on a reference plate positioned in the work plane of the LPBF machine (Fig. 2).

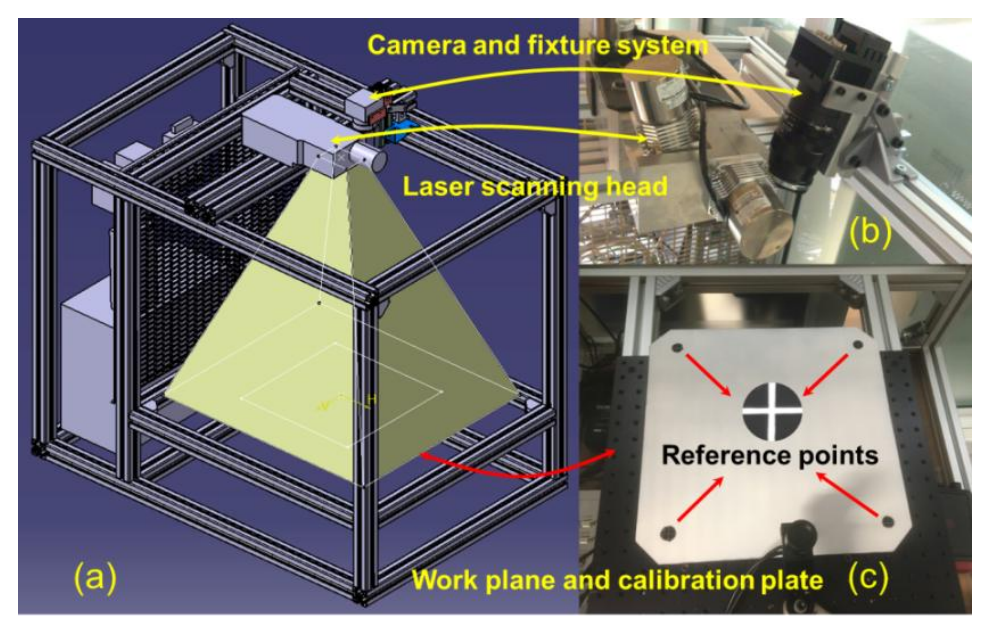

Fig. 2. (a) 3D modeling of the LBPF machine prototype used in the study, (b) high resolution monochrome camera and its fixture system installed on the machine prototype, and (c) the plate with four reference points placed on the work plane of the machine.

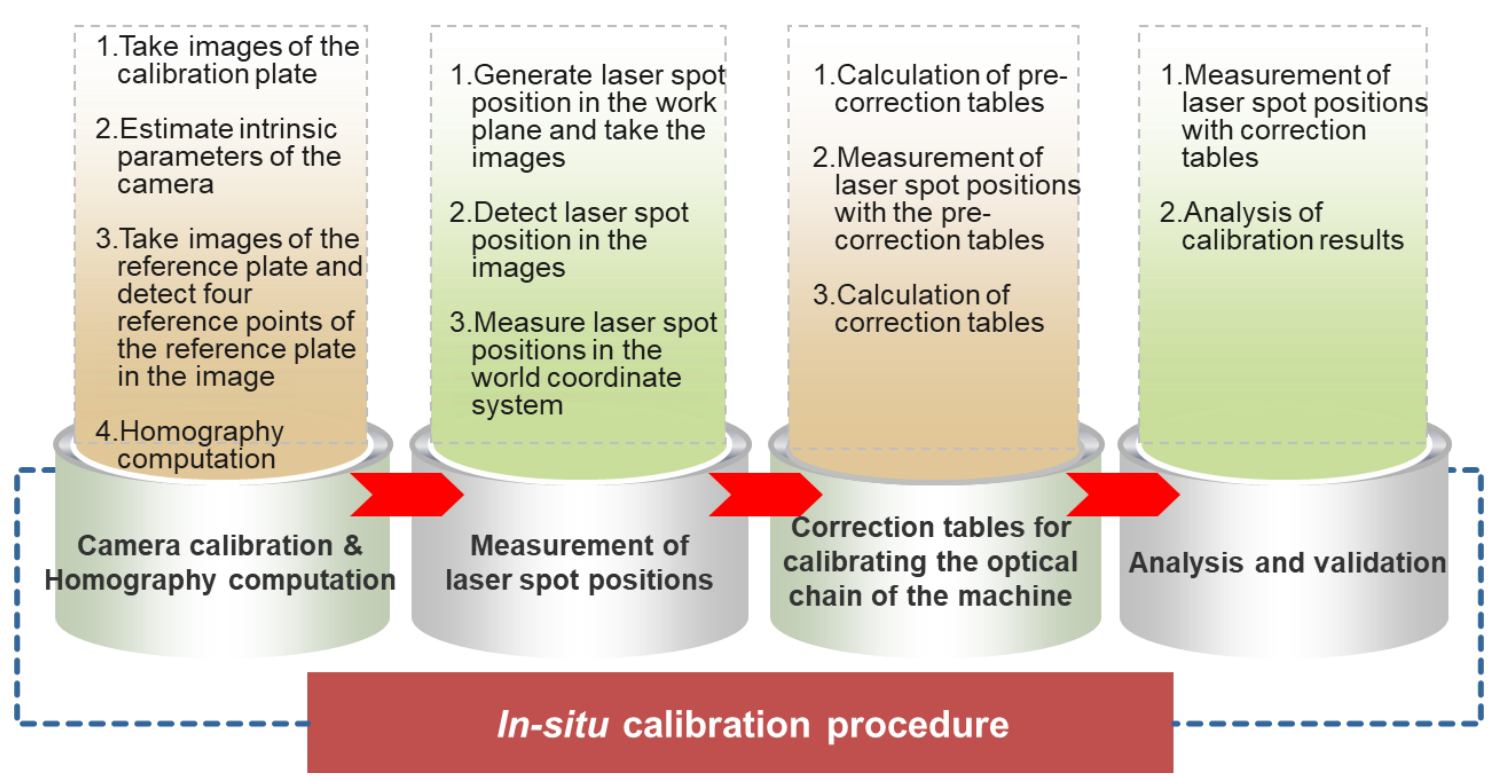

Fig. 3. The flowchart of the proposed in-situ calibration method. 
Fig. 3 presents the flowchart of the proposed calibration method:

- In the first step - "Camera calibration \& homography computation", the intrinsic parameters of the camera were estimated via the camera calibration process. These parameters were used for the image processing and the measurement method. To correct the perspective distortion of images, a homography transformation was also estimated based on four points of the reference plate (Section 4).

- In the second step - "Measurement of laser spot positions" (Section 5), before detecting the laser spot in an image, lens distortion (radial and tangential lens distortions) and perspective distortion of the image were corrected. The perspective distortion was corrected by using the homography transformation matrix estimated in the first step. The homography transformation allows an image captured in an off-axial configuration to be transformed into an orthogonal image, and the computation of world coordinates of a point from its pixel coordinates to be easily performed in an automatic manner for all positions of laser spot. The pixel coordinates of laser spot center in the corrected image were identified by using some image processing algorithms. Finally, the world coordinates (in millimeters) of laser positions in the world coordinate system were calculated by using the ratio of millimeters per pixels.

- The calibration of the optical chain of the machine was performed in two steps (Section 6): The pre-correction tables calculated from the model of the machine without defects and a set of theoretical points were used to generate the real laser spot position in the work plane. Once the positions of laser spot in the work plane were measured, they were subsequently used to identify all defects related to the geometric model of the machine and calculate the correction tables for all joint components of the optical chain. Finally, the accuracy of the proposed calibration method was analyzed.

\section{Experimental setup}

\subsection{Camera}

In this work, a monochrome camera VIEWORKS VC-71MC and a lens ZEISS DISTAGON T2.0/35 ZF were used for the measurement. The monochrome camera possesses a high resolution CMOS imaging of $10000(\mathrm{H}) \times 7096(\mathrm{~V})$ pixels. The size of each pixel is $3.1 \mu \mathrm{m}$ x $3.1 \mu \mathrm{m}$. The camera was installed on the machine according to an off-axial configuration beside the laser scanning head of the machine (Fig. 2b). This is due to the fact that the build compartment of LPBF machines requires an isolable environment from the outside of the machines, and it is filled with inert gases such as argon or nitrogen to ensure the quality of built parts and avoid the phenomena of oxidation and contamination [32]. The fixture system of the camera was designed so that the 
position and orientation of the camera can be adjusted to capture expected images of the objects on the work plane of the machine (Fig. $2 \mathrm{a}$ and $2 \mathrm{~b}$ ). Once the camera was installed on the LBPF machine prototype, the pose of the camera was fixed during the measurement.

\subsection{Reference plate}

To acquire the laser spot, a plate with four reference points was positioned in the work plane of the machine (Fig. 2c). The world coordinates of four reference points were measured a Microvue - Excel $651 \mathrm{HM}-\mathrm{HC}$ optical system. A reference point is the intersection of a vertical line and horizontal line engraved on the reference plate. The images of laser spot on the plate at different positions were captured by the camera. In order to obtain a proper image for the image processing, the exposure time of the camera was adjusted, and a light projection source was used.

\subsection{Laser source}

In the current study, a visibly red laser with a very low power was used instead of a highpower laser source as in real LBPF machines. The aim of using this laser source is to ensure the safety of operators and all experimental devices. Thereby, the reference plate can be used many times in the experiments. Moreover, with a visible laser source, the images of laser spots in the work plane captured by the camera present in common shapes such as circles or ellipses, avoiding the large deviation in the detection of laser spot centers in images. The use of a visible laser instead of a high-power laser source would also not significantly change the calibration accuracy of the machine's optical chain. This will be demonstrated at the end of section 6.3.

\subsection{Test platform}

The test platform used is composed of a 3-axis CTI (Cambridge Technology) laser scanning head and an SMC control board. It is identical to the technology used in an Addup Formup 350. The geometry of the test platform has been chosen to match the geometry of the same machine. These choices make it possible to carry out tests that can be directly transposed to an industrial machine.

\section{Camera calibration, homography computation, and measurement of 2D objects in a plane}

An image captured by the camera always presents lens and perspective distortions, which have significant effects on the accuracy of the identification of entities in the image, and subsequently on the accuracy of the measurement method. Hence, the camera should firstly was calibrated (Subsection 4.1). The calibration process aims at estimating the intrinsic and extrinsic parameters of the camera. The intrinsic parameters include the focal length, principal point, pixel skew coefficient, radial and tangential distortion coefficients, whereas the extrinsic parameters describe the pose of the camera (i.e., the camera's orientation and position). In order to detect and 
measure the laser spot position in images automatically, a homography transformation matrix based on four reference points was calculated (Subsection 4.2). Finally, the measurement method for 2D objects in the work plane was validated to ensure the quality of acquired data for the calibration process (Subsections 4.3 and 4.4).

\subsection{Camera model and camera calibration}

In computer vision, the pinhole camera model is widely used [20,33]. This model presents the mapping relation between an arbitrary $3 \mathrm{D}$ point $\boldsymbol{P}=\left(X_{w}, Y_{w}, Z_{w}\right)$ in the world coordinate system $\left(\boldsymbol{R}_{\boldsymbol{w}}\right)$ and its corresponding pixel point $\boldsymbol{p}=(u, v)$ in the pixel coordinate system $\left(\boldsymbol{R}_{u v}\right)$ of the image, as shown in Eq. (1) and Fig. 4:

$$
\lambda\left[\begin{array}{l}
u \\
v \\
1
\end{array}\right]=\left[\begin{array}{ccc}
f_{x} & s & c_{x} \\
0 & f_{y} & c_{y} \\
0 & 0 & 1
\end{array}\right] *\left[\begin{array}{ll}
\mathbf{R}_{\mathbf{3} \times \mathbf{3}} & \boldsymbol{T}_{\mathbf{3} \times \mathbf{1}}
\end{array}\right] *\left[\begin{array}{c}
X_{w} \\
Y_{w} \\
Z_{w} \\
1
\end{array}\right]=\mathbf{A}_{\mathbf{3} \times \mathbf{3}} *\left[\begin{array}{ll}
\mathbf{R}_{\mathbf{3} \times \mathbf{3}} & \boldsymbol{T}_{\mathbf{3} \times \mathbf{1}}
\end{array}\right] * \boldsymbol{P}
$$

where $\lambda$ is a non-zero scale factor; $f_{x}$ and $f_{y}$ are the effective focal length on the $u$-axis and $v$ axis of the pixel coordinate system $\left(\boldsymbol{R}_{\boldsymbol{u}}\right) ; c_{x}$ and $c_{y}$ are the coordinates of the principal point that is the intersection of the optical axis and the image plane in the image coordinate system $\left(\boldsymbol{R}_{\boldsymbol{i m}}\right) ; s$ is the skew coefficient, which is non-zero if the image axes are not perpendicular. The parameters $f_{x}, f_{y}, c_{x}$, $c_{y}$ and $s$ constitute the intrinsic parameter matrix $\mathbf{A}$ of the camera. The matrix $\mathbf{R}$ and the vector $\boldsymbol{T}$ are the rotation matrix and the translation vector between the camera coordinate system $\left(\boldsymbol{R}_{\boldsymbol{c}}\right)$ and the world coordinate system $\left(\boldsymbol{R}_{w}\right)$. They describe the extrinsic parameters of the camera.

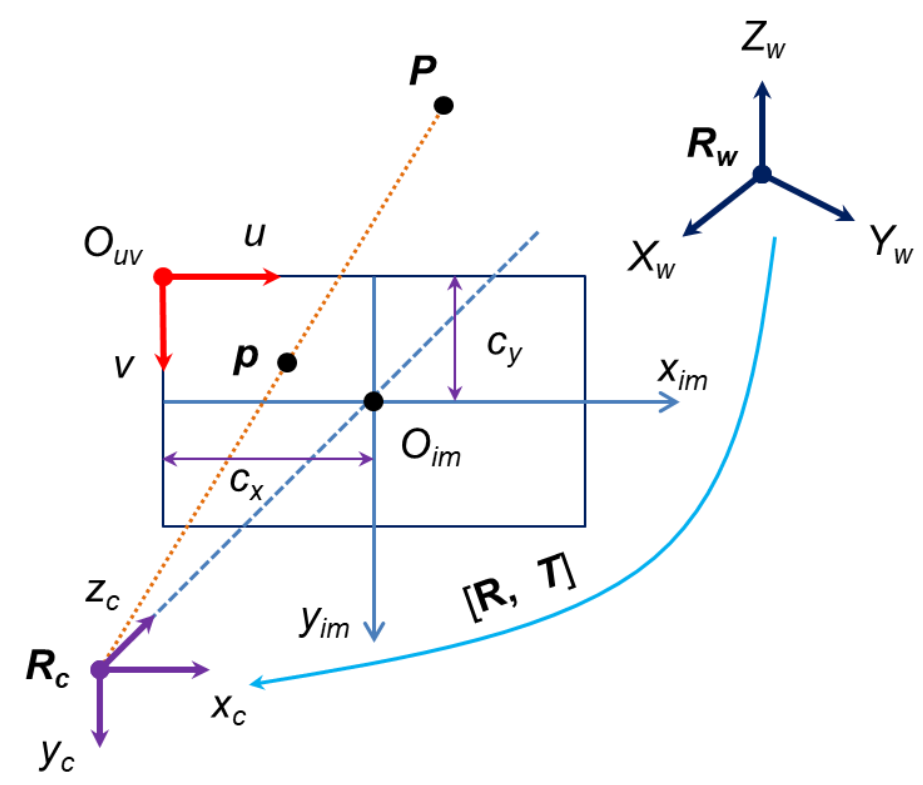

Fig. 4. Schematic diagram of the camera model. 
To present a real camera, the radial and tangential distortions of the camera lens are also taken into account. The equation describing these distortions is given by Eq. (2) [34]:

$$
\left[\begin{array}{l}
x_{\text {distorted }} \\
y_{\text {distorted }}
\end{array}\right]=\left(1+k_{1} r^{2}+k_{2} r^{4}+k_{3} r^{6}\right)\left[\begin{array}{l}
x \\
y
\end{array}\right]+\left[\begin{array}{l}
2 p_{1} x y+p_{2}\left(r^{2}+2 x^{2}\right) \\
2 p_{2} x y+p_{1}\left(r^{2}+2 y^{2}\right)
\end{array}\right]
$$

where $(x, y)$ are the coordinates of the point $\boldsymbol{p}$ in the image coordinate system $\left(\boldsymbol{R}_{i m}\right)$ without distortions: $x=u-c_{x}$ and $y=v-c_{y} ;\left(x_{\text {distorted }}, y_{\text {distorted }}\right)$ denote the coordinates of the distorted point of the point $\boldsymbol{p} ; r^{2}=x^{2}+y^{2} ; k_{1}, k_{2}$ and $k_{3}$ are radial distortion coefficients; $p_{1}$ and $p_{2}$ are tangential distortion coefficients. These distortion coefficients are also considered as intrinsic parameters of the camera.

The camera calibration aims at estimating the intrinsic and extrinsic parameters of the camera. These parameters were estimated by minimizing the cost function (3):

$$
\sum_{i=1}^{n} \sum_{j=1}^{m}\left\|\boldsymbol{m}_{i j}-\widehat{M}\left(\mathbf{A}, k_{1}, k_{2}, k_{3}, p_{1}, p_{2}, \mathbf{R}, \boldsymbol{T}, \boldsymbol{M}_{j}\right)\right\|^{2}
$$

where $\widehat{\boldsymbol{M}}$ is the projection of the world point $\boldsymbol{M}_{j}$ in the image according to Eq. (1); $\boldsymbol{m}_{i j}$ is the $j$-th image point corresponding to the world point $\boldsymbol{M}_{j}$ in the image $i ; n$ is the number of images used for the calibration process; and $m$ is the number of world points, equal to the number of image points in each image.

In this study, the camera parameters were estimated by using the "Camera Calibrator" toolbox available in Matlab and a calibration plate certificated by GOM Company with dimensions of $500 \mathrm{~mm}$ x $400 \mathrm{~mm}$ and 69 x 53 circular black markers on the white background (Fig. 5).

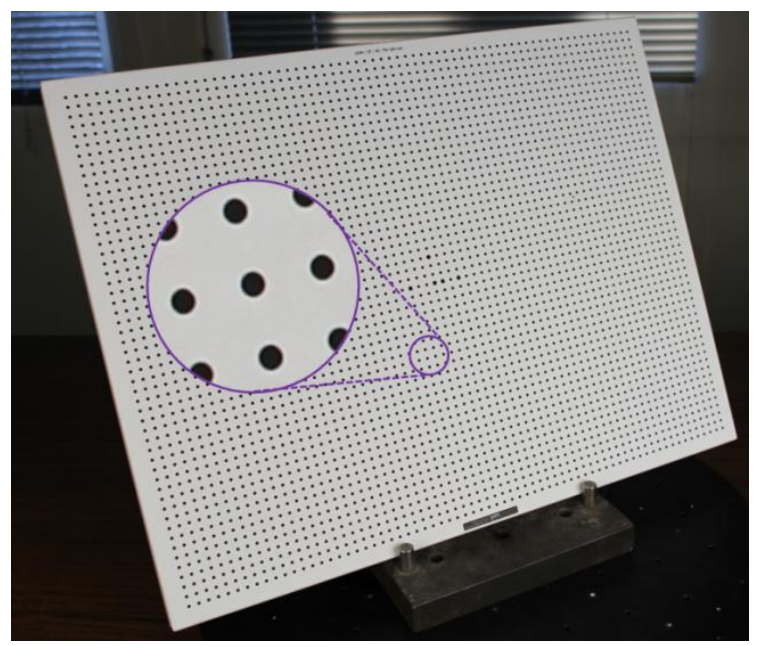

Fig. 5. Plate certificated by GOM used for the camera calibration.

In the literature, most existing camera calibration approaches [35-39] and the Camera calibrator toolbox developed in Matlab consider that the world points $\boldsymbol{M}_{j}$ must be coplanar and their 
z-coordinate is equal to 0 . In fact, the z-coordinates of marker centers of this plate present a deviation between 0 and $0.377 \mathrm{~mm}$. To take into account this flatness defect and improve the parameter determination, a least square fitting plane (LSFP) was firstly estimated from the given coordinates of all marker centers. Finally, the projections of all marker centers on the LSFP and their coordinates in the coordinate system associated with the LSFP were used as the world points for the camera calibration.

To calibrate the camera, twenty-nine images of the calibration plate captured in different positions and orientations were used. The estimated intrinsic parameters of the camera were given in Table 1.

Table 1. Estimated value of intrinsic parameters and standard deviation of the camera.

\begin{tabular}{ccccccccccc}
\hline $\begin{array}{c}\text { Intrinsic } \\
\text { parameters }\end{array}$ & $\begin{array}{c}f_{x} \\
\text { (pixels) }\end{array}$ & $\begin{array}{c}f_{y} \\
\text { (pixels) }\end{array}$ & $\begin{array}{c}c_{x} \\
\text { (pixels) }\end{array}$ & $\begin{array}{c}c_{y} \\
\text { (pixels) }\end{array}$ & $s$ (pixels) & $k_{1}$ & $k_{2}$ & $k_{3}$ & $p_{1}$ & $p_{2}$ \\
\hline Value and & 11911.16 & $\begin{array}{c}11911.83 \\
+/-\end{array}$ & $\begin{array}{c}5030.64 \\
+/-\end{array}$ & $\begin{array}{c}3593.25 \\
+/-\end{array}$ & $\begin{array}{c}0.2560 \\
+/-\end{array}$ & $\begin{array}{c}-0.0986 \\
+/-\end{array}$ & $\begin{array}{c}0.1171 \\
+/-\end{array}$ & $\begin{array}{c}-0.0410 \\
+/-\end{array}$ & $\begin{array}{c}-0.0002 \\
+/-\end{array}$ & $\begin{array}{c}0.0004 \\
+/-\end{array}$ \\
the standard & $\begin{array}{c}+/- \\
\text { +/- }\end{array}$ & 0.2756 & 0.1240 & 0.1139 & 0.0127 & 0.0001 & 0.0011 & 0.0039 & 0.0000 & 0.0000 \\
\hline
\end{tabular}

It can be seen that the values of the tangential distortion coefficients ( $p_{1}$ and $\left.p_{2}\right)$ are very small, which shows the quality of the optics. Thus, the influence of tangential distortion can be negligible. The standard deviations of $f_{x}, f_{y}, c_{x}, c_{y}$ and $s$ are fewer than 0.3 pixels, meaning a good uncertainty in the estimation of camera parameters. On the other hand, the radial distortion coefficients $\left(k_{1}\right.$ to $\left.k_{3}\right)$ are noticeable and have significant effects on the identification precision of expected entities in the images. Therefore, this distortion should be corrected before the detection of laser spot centers.

\subsection{Homography computation}

The perspective distortion always exists in an image taken in a non-orthogonal configuration. This distortion can be corrected by estimating a 2D homography matrix $\mathbf{H}$ defined as Eq. (4) [40]:

$$
\left[\begin{array}{c}
u^{\prime} \\
v^{\prime} \\
1
\end{array}\right]=\mathbf{H}_{3 \times 3}\left[\begin{array}{l}
u \\
v \\
1
\end{array}\right]
$$

where $(u, v)$ are the coordinates in the initial image and $\left(u^{\prime}, v^{\prime}\right)$ are the coordinates in the orthogonal image. Herein, the matrix $\mathbf{H}$ was estimated by using four correspondences (A, B, C, D) $\leftrightarrow\left(A^{\prime}, B^{\prime}, C^{\prime}, D^{\prime}\right)$, as shown in Fig. 6a.

The points (A, B, C, D) in the initial image - each point is the intersection of a vertical line and a horizontal line engraved on the plate, were detected by using the Algorithm 1 (described in 
subsection 4.3), whereas the points (A', B', C', D') in the orthogonal configuration were defined from the corresponding world points, the focal length and principal point of the camera.

By using the perspective warping with the homography transformation matrix $\mathbf{H}$, the perspective distortion of the initial image was effectively corrected, and the corrected image appears as an image captured in the orthogonal configuration of the camera (Fig. 6b).

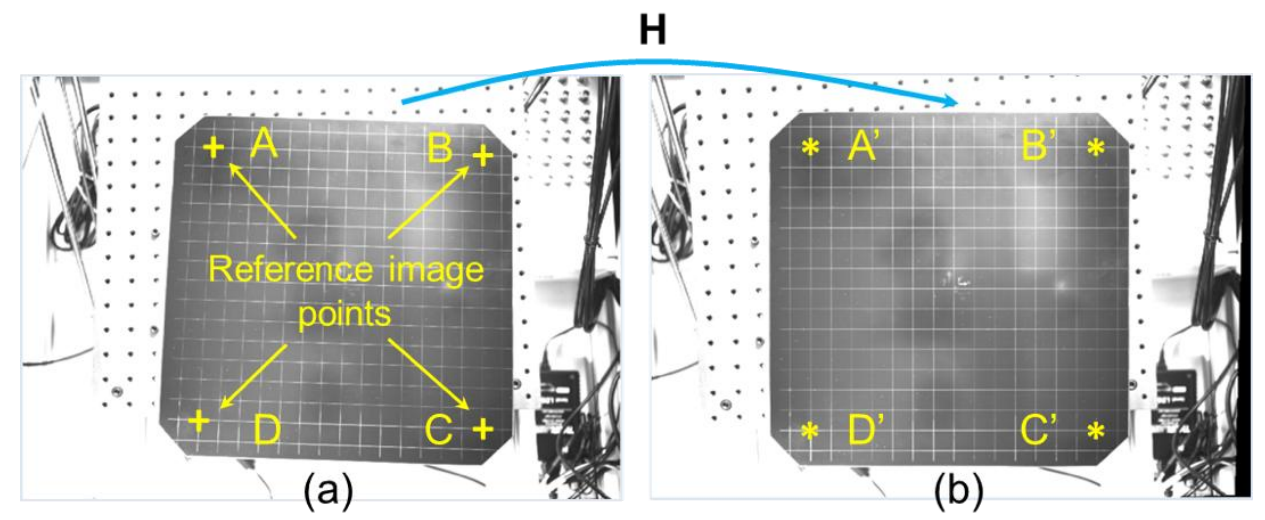

Fig. 6. Perspective distortion correction by applying a homography transformation: (a) initial image with perspective distortion and (b) image after the perspective warping with the homography $\mathbf{H}$.

\subsection{Measurement method of $2 D$ objects in a plane}

4.3.1. Detection of a grid point in the image

In this work, the interested $2 \mathrm{D}$ objects were the grid points engraved in the reference plate. The pixel coordinates of a gird point in the image were determined through two steps: $(i)$ correct lens distortion of the image, and (ii) identify the intersection points of the gird on the image. The lens distortion correction was easily performed by using the "camera calibrator" toolbox of Matlab software.

In the image, each line of the grid is characterized by a bright line with a width of about ten pixels. Each point of the grid is the intersection of a vertical line and a horizontal line. Hence, a grid point in the image was identified by the Algorithm 1 developed in Matlab, as follows:

\footnotetext{
Algorithm 1: Identification of a grid point in the image

1. Crop a square region of interest (RoI) around the interested point to isolate it from the total image and reduce the image processing time (Fig. 7).

2. In the cropped image, detect the points on boundaries of the vertical and horizontal lines by using the CANNY algorithm [40]. Sometimes it also detects boundary points of bright regions in the RoI (Fig. 7b).

3. From the detected boundary points, apply the RANSAC algorithm [40] to classify and
} 
construct two vertical lines (green) and two horizontal lines (yellow), which are the boundary lines corresponding to the vertical and horizontal lines.

4. Calculate four intersections (x, red) of four constructed lines and their gravity centroid (*, violet). This gravity centroid was considered as the grid point needs to identify (Fig. $7 b)$.

5. Transform the pixel coordinates of the identified grid point in the cropped image (Fig. 7b) into the pixel coordinate system of the corrected image (Fig. 7a)

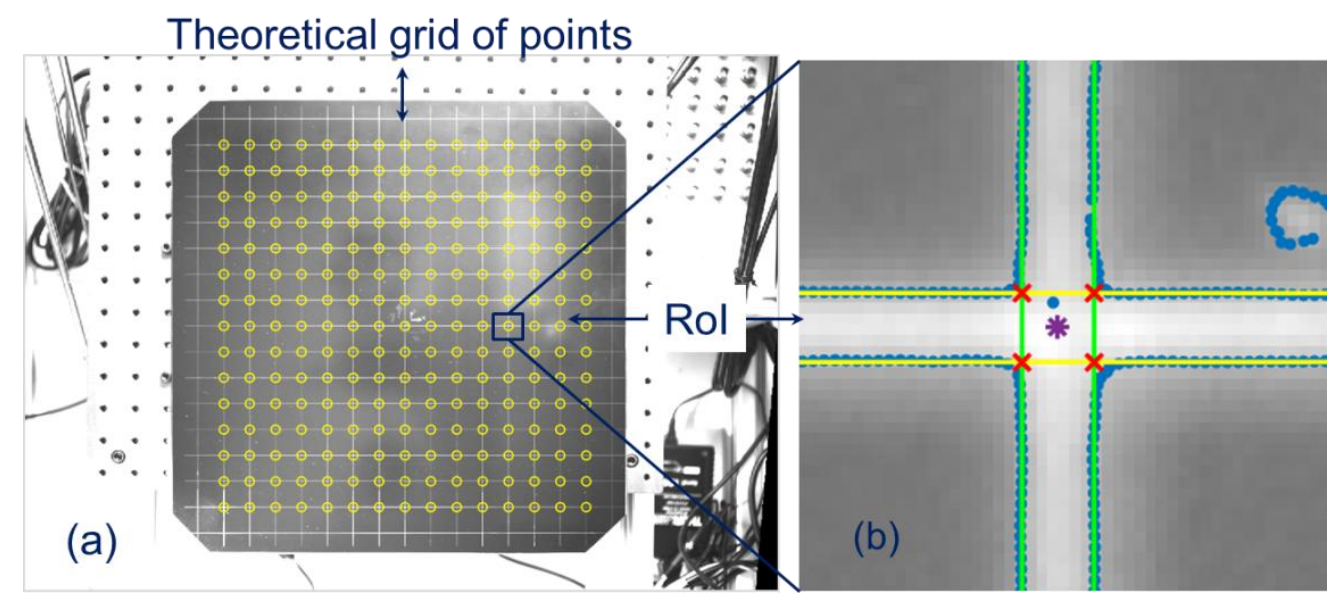

Fig. 7. Identification of grid points in the corrected image: (a) creating a theoretical grid of points and (b) determining a point of the grid (intersection of two lines) in the image.

The algorithm 1 was used to detect four reference points (Fig. 2c), which are intended for the homography computation, and detect all grid points on the plate (Fig. 7a) for the validation of the measurement method.

4.3.2. Detection of all grid points in the image for the measurement method

In order to detect all grid points engraved on the plate automatically, the correction of lens distortions and perspective distortion was firstly performed. Thereafter, a grid of theoretical points was created from four points (A', B', C', D') (Figs. 6b and 7a) - each theoretical point is very close to the grid point to be detected. Finally, all grid points in the corrected image were automatically detected by the Algorithm 1.

\subsubsection{Computation of the world coordinates of grid points}

To calculate the coordinates of the grid points in the world coordinate system $\left(\mathbf{R}_{\mathbf{w}}\right)$, the ratio $(r)$ between a distance in the world coordinate system $\left(\mathbf{R}_{\mathbf{w}}\right)$ per the corresponding distance in the orthogonal image was used. This ratio was defined as Eq. (5):

$$
r=\frac{\left\|\overrightarrow{\boldsymbol{P}_{J} \boldsymbol{P}_{k}}\right\|}{\left\|\overrightarrow{\boldsymbol{p}_{J} \vec{p}_{k}}\right\|}(\mathrm{mm} / \text { pixel })
$$


where $\boldsymbol{p}_{\boldsymbol{j}}, \boldsymbol{p}_{\boldsymbol{k}}$ are the image points selected from four reference points (A', B', C', D') in the orthogonal image (Fig. 7b) and $\boldsymbol{p}_{j} \neq \boldsymbol{p}_{\boldsymbol{k}} ; \boldsymbol{P}_{\boldsymbol{j}}$ and $\boldsymbol{P}_{\boldsymbol{k}}$ are respectively the corresponding world point of $\boldsymbol{p}_{j}$ and $\boldsymbol{p}_{\boldsymbol{k}}$.

Finally, the coordinates of the grid points measured by the proposed method were calculated as Eq. (6):

$$
\boldsymbol{P}_{\text {mesure }}=r^{*}(\boldsymbol{p}-\boldsymbol{C})
$$

where $\boldsymbol{C}=\left(c_{x}, c_{y}\right)$ is the principal point of the camera, $\boldsymbol{P}_{\text {mesure }}$ is the grid point measured in the world coordinate system in millimeters, and $\boldsymbol{p}$ is the corresponding image point in the pixel coordinate system of the orthogonal image.

\subsection{Validation of measurement method}

Fig. 8 shows the position errors between the grid points measured by the proposed method and the reference points given by the manufacturer of the plate. For all measured points, the position errors are less than $0.058 \mathrm{~mm}$, and the mean error is approximately $0.028 \mathrm{~mm}$. In the error histogram (Fig. 8 on the right), it is found that about $82 \%$ of measured points have errors less than $0.04 \mathrm{~mm}$. Only $8 \%$ of measured points present errors between $0.045 \mathrm{~mm}$ and $0.058 \mathrm{~mm}$.
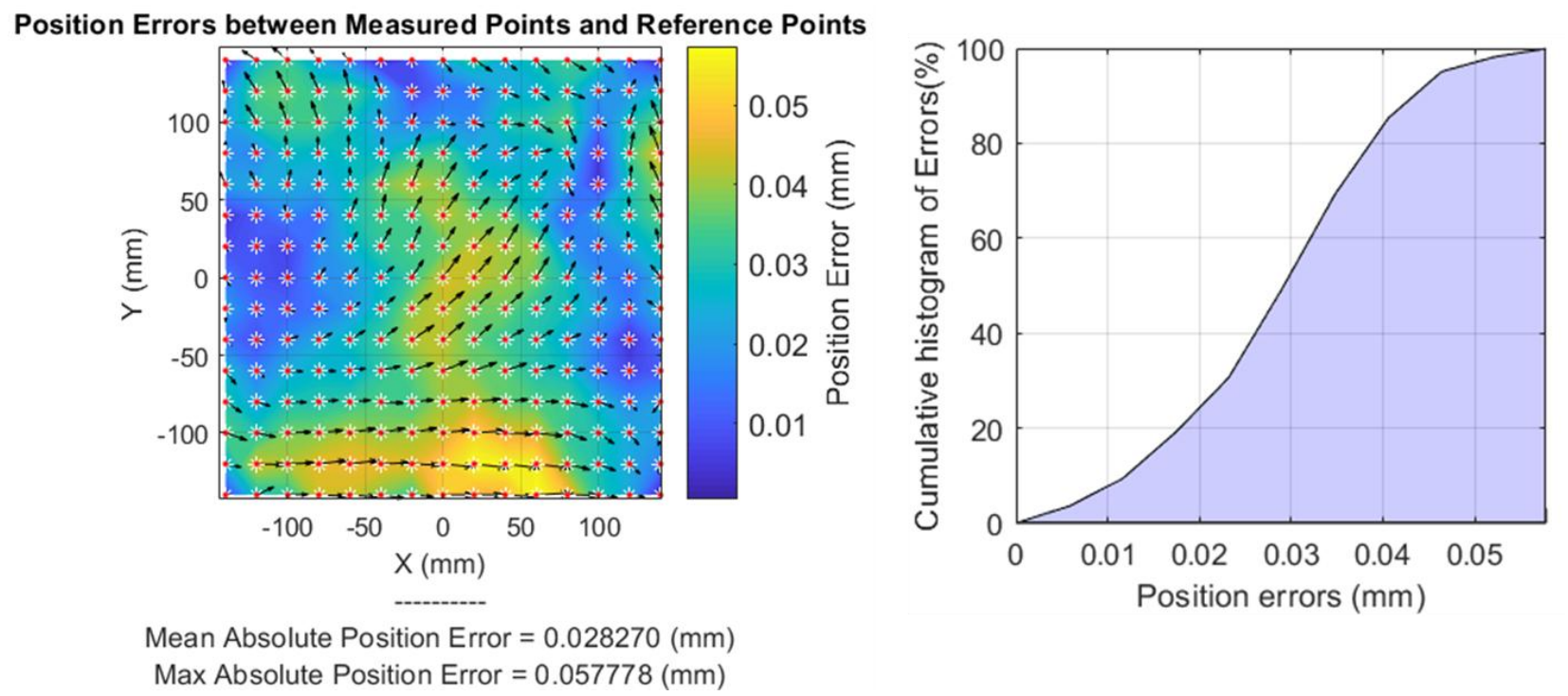

Fig. 8. Distribution of position errors between the measured points (red) and the reference points (white) and the cumulative histogram of position errors.

It is also observed that the distribution of errors is not homogeneous. This phenomenon can be explained by the inhomogeneity of the grid lines engraved on the plate, and the image-taking condition (for example, the light intensity and reflection of light on the plate). This produces the lines in the image with different levels of clarity. In fact, the accuracy of the proposed measurement method greatly depends on the accuracy of the homography calculation and the grid point detection. 
Therefore, the detection of grid points in the images plays an important role. The quality of the detection of grid points in the images strongly depends on the brightness of the lines in the image. This problem can be eliminated by improving and adjusting the image-taking condition and selecting the proper threshold values for detecting boundary points of lines in the CANNY algorithm (Fig. 8b).

In summary, the results shown in this section confirm that the proposed method can detect image points in the image correctly, and the points in the work plane of the machine can be efficiently measured with an average position error lower than $0.03 \mathrm{~mm}$. Therefore, the method can be used for the in-situ calibration of the optical chain of LPBF machines.

\section{Measurement of laser spot positions in the work plane}

\subsection{Shape of laser spot}

In the experiment, the reference plate was used to acquire the laser spot shape. This plate was positioned on the work plane of the machine so that the axis $X_{p}$ and $Y_{p}$ of the plate coordinate system $\boldsymbol{R}_{\boldsymbol{p}}$ are respectively parallel to the axis $X_{w}$ and $Y_{w}$ of the world coordinate system $\boldsymbol{R}_{w}$ of the work plane of the machine (Fig. 9). The images of laser spot in the reference plate were captured by the camera.

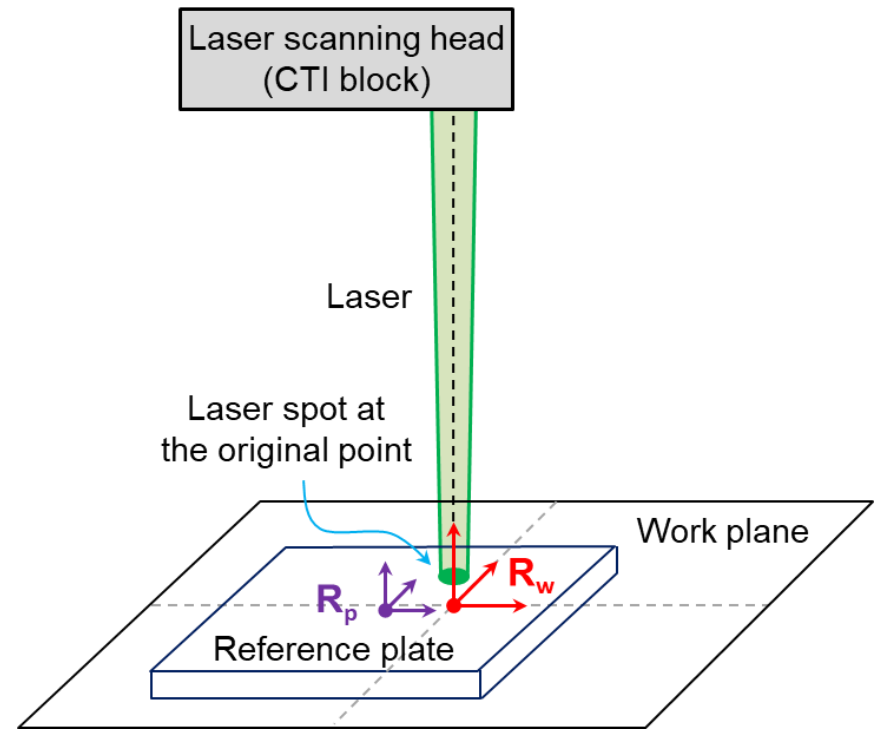

Fig. 9. Illustrating the positioning of the reference plate on the work plane of the machine.

In images, a laser spot is ideally represented by a small region of bright and white pixels and the boundary of the laser spot is a circle or an ellipse. However, depending on many factors such as the firing angle of the laser, the surface state, the surface material, the light of the working environment, the resolution of the camera, the exposure time of the camera, and so on, the actual laser spot form is often elliptical (Fig 10a) or irregular (Fig 10b). In addition, the laser spot images 
present some types of noises, for example, background noise (Fig. 10c), photon noise, and readout noise (Fig. 10d) [41]. To limit reflections and reduce noise on the image, the surface of the plate was covered with a mat material except four reference points (Fig. 2c) that were used to calculate the homography in the measurement method. After that the background noise was eliminated (Fig. 10a).
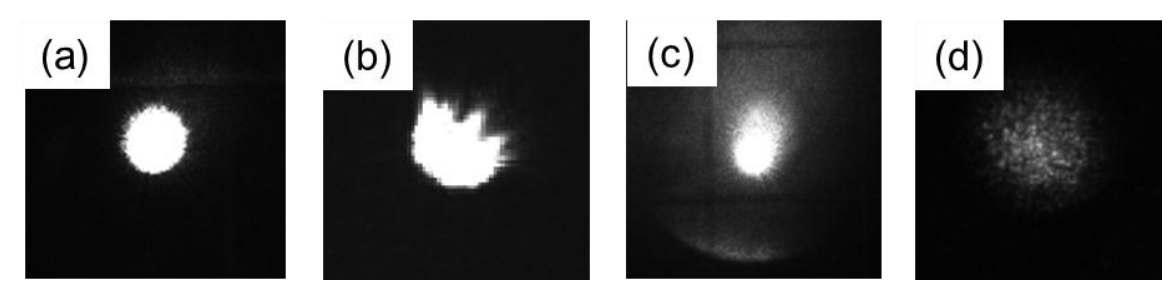

Fig. 10. Various shapes of the laser spot appearing in the image: (a) ellipse, (b) irregular shape, (c) the laser spot with the background noise, and (d) the laser spot with readout noise.

\subsection{Detection of laser spot in the images}

The steps to detect the laser spot center were shown in Fig. 11. Firstly, the image of the laser spot was corrected in terms of lens and perspective distortions. In addition, a square region of interest (RoI) including the laser spot was cropped to isolate the laser spot from the total image and reduce the image processing time.

To reduce the photon and readout noises while leaving the edges of the laser spot unchanged, some image filtering approaches can be used. Several algorithms are frequently used to filter images, such as adaptive median filtering, Gaussian filtering, medium filtering, and Wiener filtering [42]. In this work, to reduce the photon and readout noises while leaving the edges of the laser spot unchanged, the 2D Gaussian filtering [37,43] was employed. Thereafter, the filtered image was converted into a binary image using a threshold value defined as $T s=I_{\max } / 1.1$ [44], where $I_{\max }$ is the maximum brightness in the image.

There are two groups of methods in the literature for detecting the center of the laser spot:

(i) In the first group, the contour of the laser spot was firstly detected, and the center of the laser spot was then determined by estimating a circle or an ellipse from the detected contour by the least square method [44-46]. These methods are effective for detecting laser spots with a relatively large size and a regular shape (e.g. circle or ellipse). 


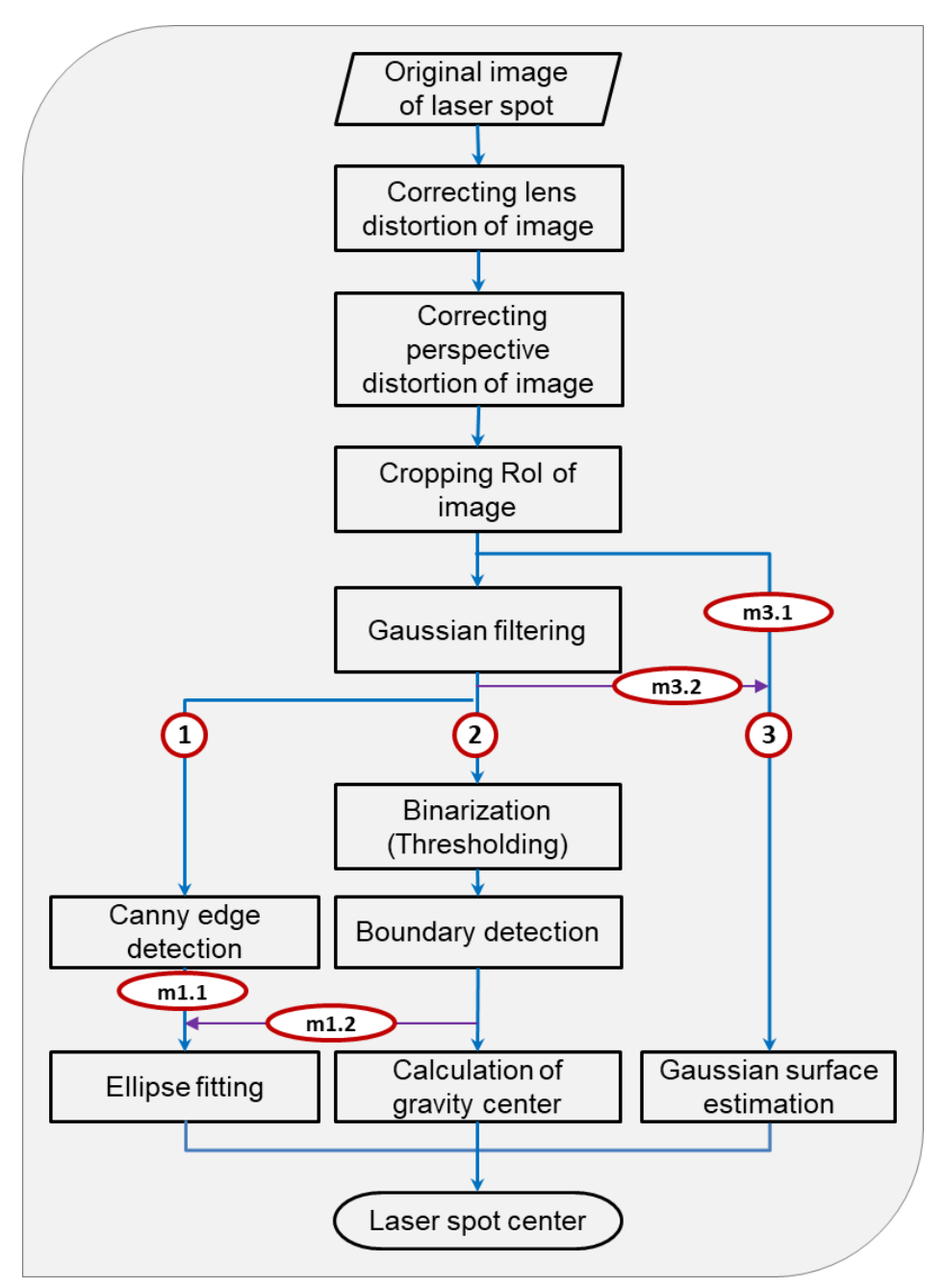

Fig. 11. Different methods of laser spot center detection.

(ii) The second group of methods was based on analyzing the characteristics of the laser spot area as a function of pixel intensity of the image, for example, Gaussian quadric estimation [47] and center of gravity [41,48-52]. The center of the light spot is generally located at the top of the intensity surface. Hence, these methods are more suitable for determining the center of the laser spot, in particular for well-structured laser spots.

In this work, three methods were considered to detect the position of the laser spot, namely ellipse fitting (1), center of gravity (2), and Gaussian quadric estimation (3), as depicted in Fig. 11. (m1.1) is the method (1) using the input data detected by the CANNY algorithm [40], while (m1.2) is the method (1) using the input data detected by the binarization. In the same way (m3.1) is the method (3) using the input image without Gaussian filtering, and (m3.2) is the method (3) using the input image filtered by the Gaussian filtering operation.

Table 2. Images of laser spot simulated by the super-Gaussian distribution function without noises and with white Gaussian noises. 


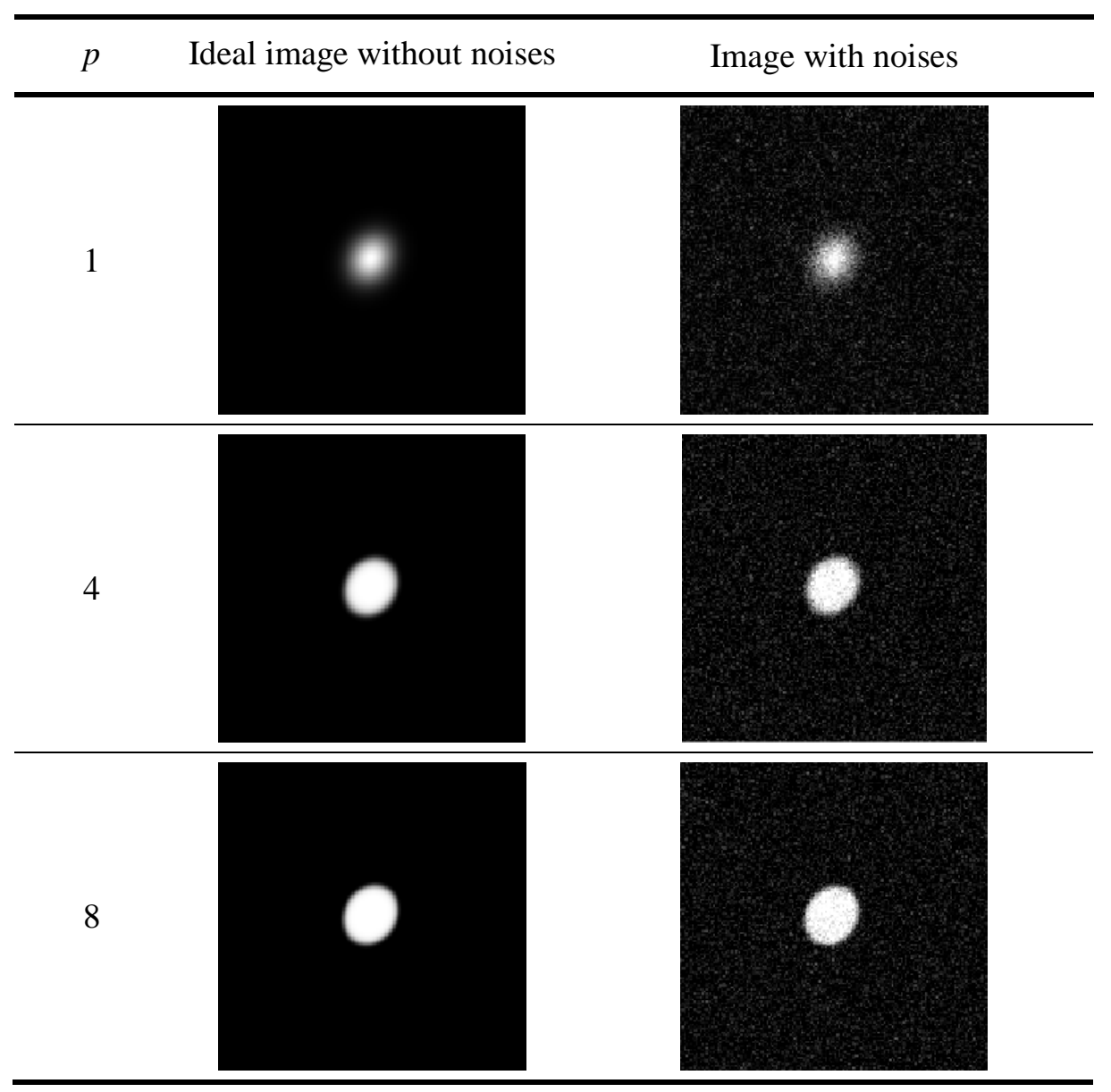

\subsubsection{Influence of the noise}

In order to evaluate the accuracy and performance of these methods, three cases of laser spots were simulated by using a super-Gaussian distribution function of order $p$ [53-55], as shown in Table 2. These images were representative for certain actual shapes of laser spot on the reference plate taken by the camera. The size of each image is $160 \times 160$ pixels and the center of laser spot for all ideal images without noises is the image center $\left(x_{0}, y_{0}\right)=(80,80)$ (pixels). Fig. 12 presents the detection error between the laser spot center detected by the considered methods and that of the ideal image center $\left(x_{0}, y_{0}\right)$. It is revealed that the methods $(\mathrm{m} 1.2),(\mathrm{m} 2)$, and $(\mathrm{m} 3.2)$ are more stable and the detection error is less than 0.3 pixels in the three study cases. 


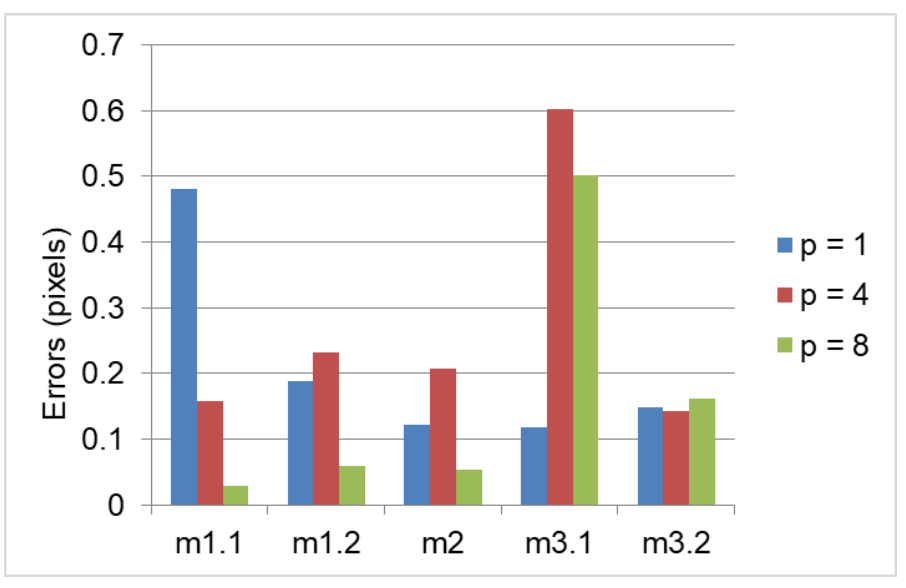

Figure 12. Detection errors caused by different methods.

\subsubsection{Influence of the set up environment}

Due to the vibration of the machine and the change of light intensity of the experimental room, the position of an actual laser spot is also unstable. Fig. 13 shows the positions of an actual laser spot detected by the methods (m1.2), (m2), and (m3.2) in 20 images taken successively by the camera. For the detection methods $(\mathrm{m} 1.2)$ and $(\mathrm{m} 2)$, the maximum laser spot variation distance in the images is 0.26 and 0.27 (pixels), respectively, whereas the method (m3.2) shows the maximum laser spot variation distance of 0.90 (pixels). This observation demonstrates that the methods (m1.2) and $(\mathrm{m} 2)$ allow us to obtain more stable detection results of the real laser spot center.

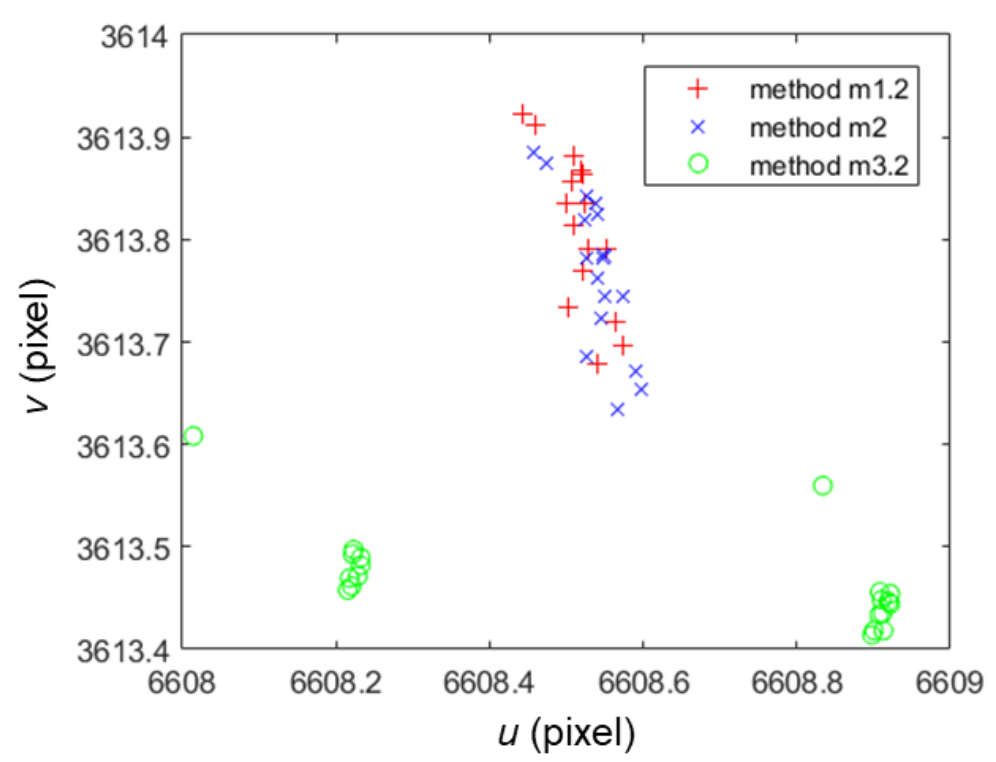

Figure 13. Influence of system vibration on measurement accuracy.

In terms of the image processing time, the method $(\mathrm{m} 2)$ is more rapid. Thus, the method (m2) - Center of gravity was finally taken into account for the study.

\subsection{Determination of laser spot positions in the world coordinate system}


Once the laser spot center in the cropped image was detected, its coordinates were transformed into the pixel coordinate system of the corrected initial image, and the coordinates of laser spot position in the world coordinate system of the plate were determined as presented in Subsection 4.3.3.

\section{In-situ calibration of galvanometric scanning head for a LPBF machine prototype}

\subsection{Model of LBPF machines}

In this study, the models developed by Godineau et al. [7] for the optical chain of industrial SLM machines - i.e. forward kinematic model (FKM) and inverse kinematic models (IKM), and the proposed measurement method (Section 2) were used for calibrating the optical chain of an LPBF machine prototype installed in our laboratory (Fig 2.a). The forward kinematic models (FKM) expresses the coordinates of the laser spot position in the work plane $\boldsymbol{X}=(x, y)$ as a function of the joint commands $\boldsymbol{Q}=\left(\theta_{x}, \theta_{y}\right)$ (Fig. 1 on the left) and assembly defects $\boldsymbol{\delta}: \boldsymbol{X}=f(\boldsymbol{Q}, \boldsymbol{\delta})$, while the inverse kinematic models (IKM) allows determining the joint commands $\boldsymbol{Q}$ and assembly defects $\boldsymbol{\delta}$ from $\boldsymbol{X}$ [7].

The LPBF machine prototype considered in this work possesses the same optical chain (i.e. the laser galvanometric scanning head) to that of the industrial LPBF machine. However, the CTI block of mirrors of the prototype machine has only a protective window, whereas the CTI block of the industrial machine possesses two protective windows (Fig. 1). Thus, the model of the optical chain in this study was adapted from the model in [7] by removing the protective window 2 of the CTI block (Fig.1) and two related defects. The model in this study has 28 assembly defects instead of 30 defects as in [7]. Moreover, in this study, we used the reference plate (Fig. 2c) positioning on the work plane of the machine to measure the laser spot position in the step of experimental measurement phase. Thus, the work distance from the CTI head and the work plane must be modified by subtracting the thickness of the plate. This means the work plane considered in this study is the top surface of the reference plate.

\subsection{Calibration procedure}

The calibration process of the laser galvanometric scanning head consists of two steps, which are detailed in the next two sections.

\subsubsection{Calculation of pre-correction tables}

In the first step, the pre-correction tables/matrix for all joint commands were calculated from the IKM without defects and a gird of theoretical laser spot positions in the work plane - $\boldsymbol{X}_{t h}\left(x_{t h}, y_{t h}\right)$. The theoretical positions of laser spot consist of $65 \times 65$ points in a calibration region of $560 \mathrm{~mm} x$ 
$560 \mathrm{~mm}$. The pre-correction tables/matrix $\left(\left[\Delta \mathbf{x}_{\text {pre }}\right]_{65 \times 65}\right.$ and $\left.\left[\Delta \mathbf{y}_{\text {pre }}\right]_{65 \times 65}\right)$ in bits were calculated according to the schema presented in Fig. 14.



Fig. 14. Calculation of pre-correction tables for joint commands.

\subsubsection{Calculation of correction tables}

In the second step, the correction tables were calculated from the experimental data and the IKM with identified assembly defects, as shown in Fig. 15. Firstly, the pre-correction tables were integrated in the ScanMaster Controller (SMC) of the controlling device. A subset of theoretical points $\boldsymbol{X}_{\boldsymbol{t h}}^{*}$ extracted from the theoretical gird of points $\boldsymbol{X}_{\boldsymbol{t h}}$ was used to generate real positions of laser spot in the top surface of the reference plate by using the ScanMaster Designer software. At each time the laser projected in the reference plate surface, its spot was captured by the camera. Subsequently, all captured images were used as the input for the measurement method of laser spot positions, as described in Section 5. The obtained measuring data $\boldsymbol{X}_{\boldsymbol{e x p}}$ was then used for the identification of 28 assembly defects $\delta$ by using the algorithm developed in [7]. Finally, the correction tables were calculated in the same manner for calculating the pre-correction tables, but using the IKM with already identified defects.

\subsubsection{Data acquisition}

Due to the dimensions of the reference plate are limited in $(350 \mathrm{~mm}$ x $350 \mathrm{~mm})$, the measurement of laser spot positions was performed at $19 \times 11$ positions. These positions of laser spots were generated by shooting the laser according to the order of theoretical grid points $\boldsymbol{X}_{\boldsymbol{t h}}^{*}$ (209 points), as shown in Fig.16. 


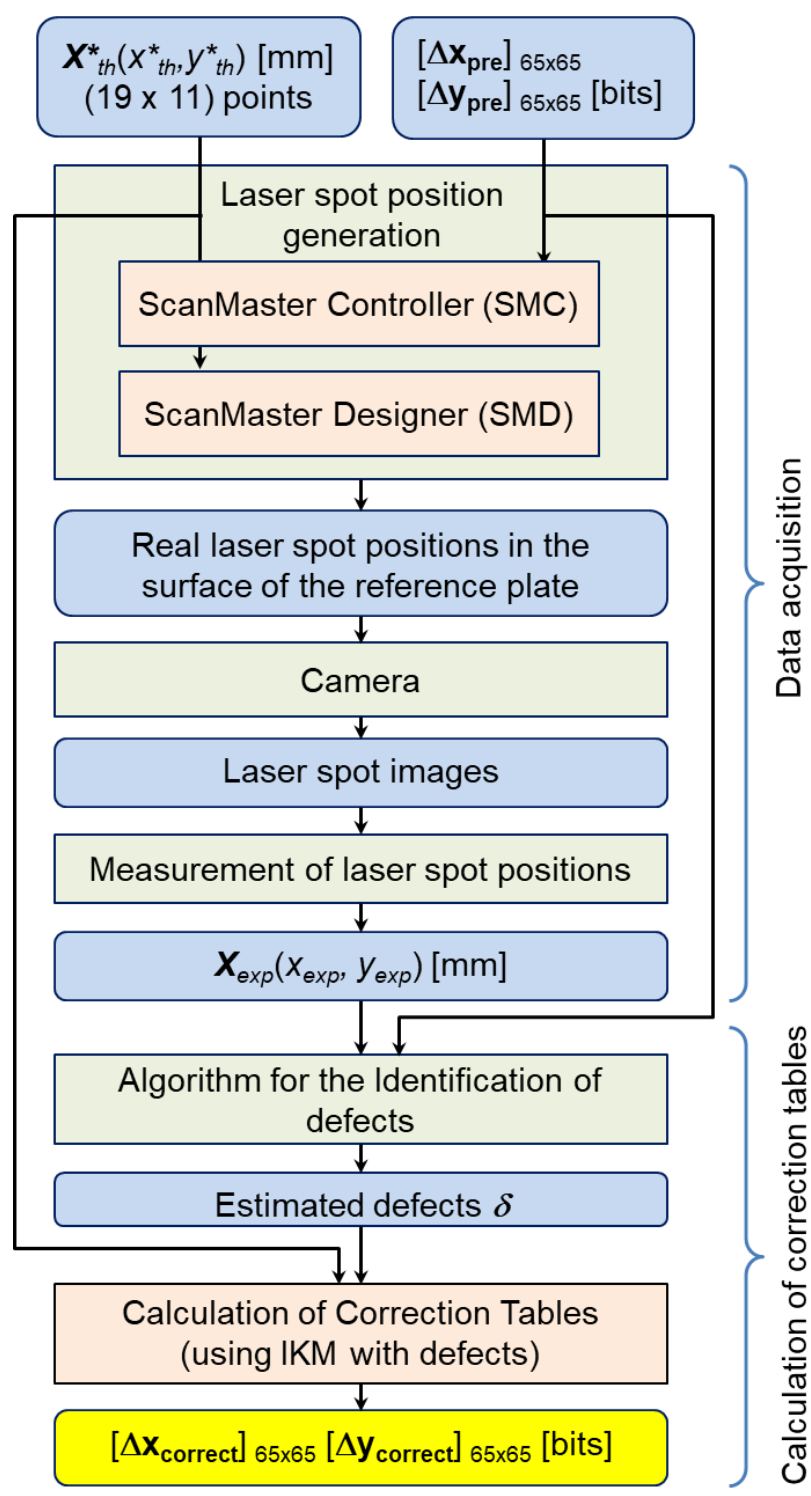

Fig. 15. Calculation of correction tables for joint commands.

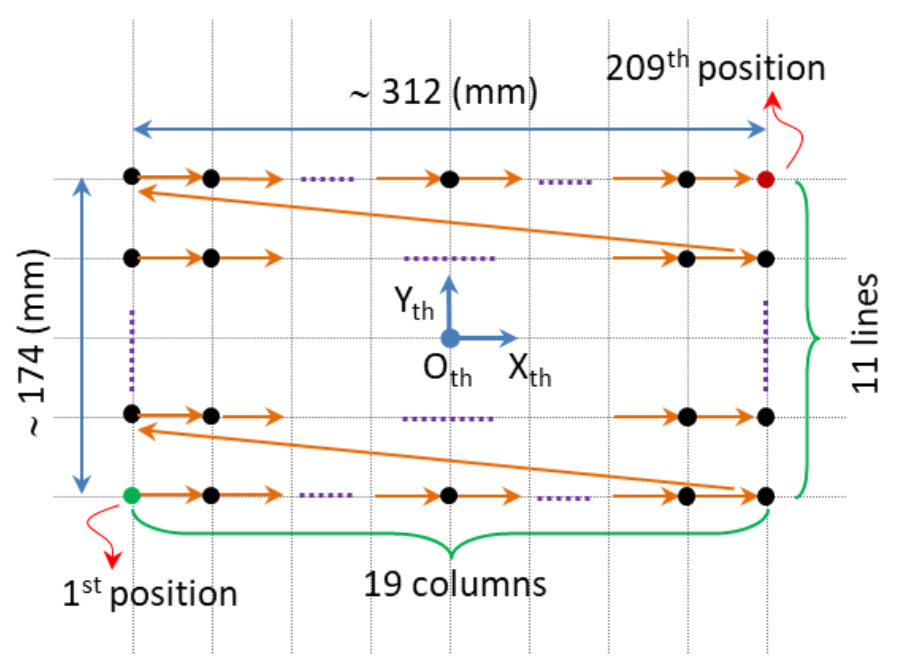

Fig. 16. Order for laser shooting and laser spot positions to be measured. 
In the experiment, the positions of laser spot were measured in the world coordinate system of the reference plate $\left(\boldsymbol{R}_{\boldsymbol{p}}\right)$. Hence, it is necessary to measure the position of laser spot corresponding to the origin of the world coordinate system of the machine $\left(\boldsymbol{R}_{w}\right)$ in $\left(\boldsymbol{R}_{\boldsymbol{p}}\right)$ (Fig. 9). Denote $\boldsymbol{X}_{\text {mes }(R w)}^{0}$ is the origin of the world coordinate system of the machine $\left(\boldsymbol{R}_{\boldsymbol{w}}\right)$ in $\left(\boldsymbol{R}_{\boldsymbol{p}}\right)$-and $\boldsymbol{X}_{\text {mes (Rw) }}^{i}$ is the position of the laser spot measured in the world coordinate system of the reference plate $\left(\boldsymbol{R}_{\boldsymbol{p}}\right)$, the position of the laser spot in the world coordinate system of the machine $\boldsymbol{X}_{\exp (R w)}$ were determined by Eq. (7):

$$
\boldsymbol{X}_{\text {exp }}^{i}=\boldsymbol{X}_{\text {exp (Rw) }}^{i}=\boldsymbol{X}_{\text {mes }(R p)}^{i}-\boldsymbol{X}_{\text {mes }(R w)}^{0}, i=1 \ldots 209
$$

\subsection{Calibration results and discussion}

Fig. 17 shows the deviation between the measured positions (red) and those of the model without defects (white). It is found that position error values are still important in compared to the requirement of the manufacturer. The average value of position errors is about $0.39 \mathrm{~mm}$ and the maximum value is about $1 \mathrm{~mm}$.

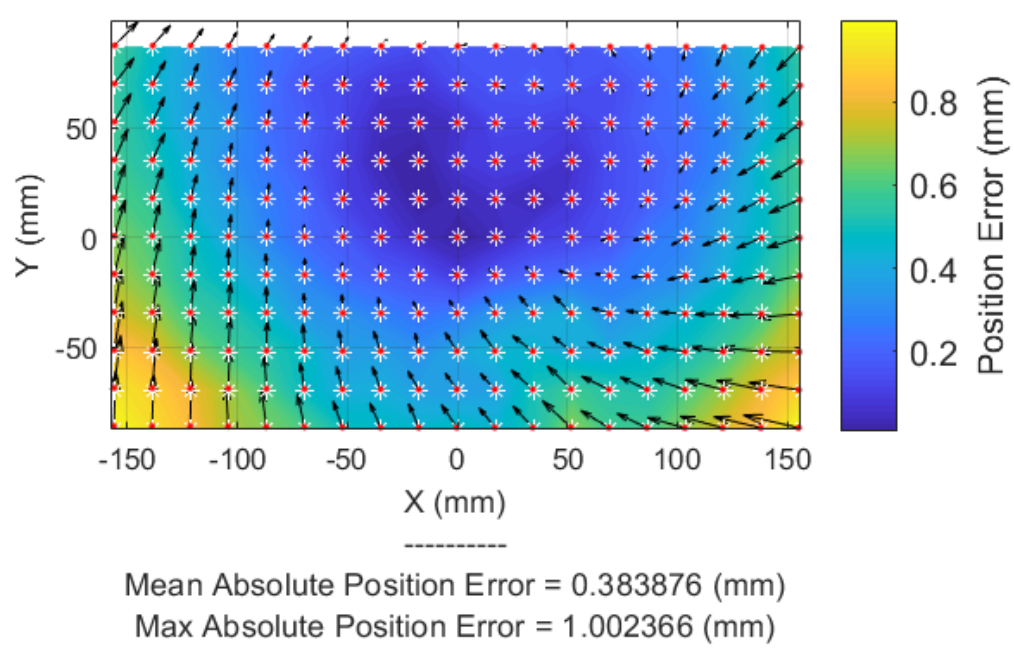

Fig. 17. Distribution of position errors between the measured positions (red) and those of the model without defects (white).

To evaluate the accuracy of the calibration, the same steps in the data acquisition presented in Fig.15 was repeated. However, in this case, the correction tables were used instead of the precorrection tables. The measured positions of actual laser spot were then compared with those generated by the identified model of the machine. Fig. 18 presents the position error distribution between the measurement and the identified model with defects of the machine. It reveals that the position errors between the measured positions and those generated by the identified model are very small with an average error of $0.03 \mathrm{~mm}$. The maximum position error is less than $0.075 \mathrm{~mm}$. There are approximately $80 \%$ of positions with an error less than $0.04 \mathrm{~mm}$ (Fig. 19). 


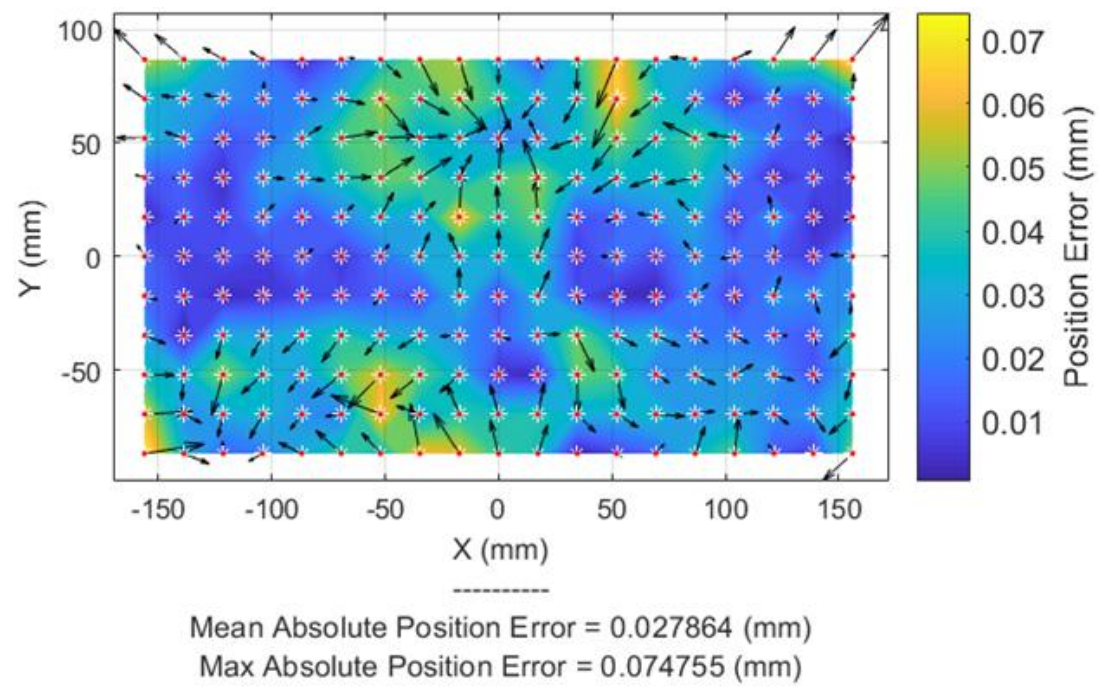

Fig. 18. Distribution of position errors between the measured laser spot positions (red) and those of the identified model (white) after the calibration.

The distribution of position errors was not homogeneous. In fact, the laser spot shape varies according to the laser shooting positions and the conditions of taking the images, resulting in the deviation of laser spot positions in the images.



Fig. 19. Cumulative histogram of position errors from Fig. 18.

In comparison with the model without defects of the machine (i.e., the model using the precorrection tables), after the calibration process the average position error was significantly reduced from $0.38 \mathrm{~mm}$ (Fig. 17) to $0.03 \mathrm{~mm}$ (Fig. 18). The maximum error was also reduced from $1.00 \mathrm{~mm}$ to $0.075 \mathrm{~mm}$.

In comparison with the calibration results obtained in [7] for an industrial LPBF machine, which uses the same laser scanning head as the machine prototype in this study, the residual errors 
between the measurement and the identified model of the machine were higher. In [7], after the calibration process, $95 \%$ of positions in the calibration zone presents an error less than $0.02 \mathrm{~mm}$, and the maximum error is below $0.025 \mathrm{~mm}$. The calibration results in this study were still not good as those obtained in [7]. This can be explained by some following reasons:

- The difference between the geometry of the machine prototype used in this study and the model developed in [7] for a real industrial machine. The calibration and measurement results are also affected by the vibration of the machine structure, the variation of light during measurements.

- The shape of the laser spot greatly varies over all the studied points. Thus, detection quality criterion should be defined in order to eliminate outliers.

- The area used for calibration in our case is limited by the size of the plate. Theoretically, the calculation of the correction tables/matrix with dimensions of $65 \times 65$ requires $65 \times 65$ measured points. However, the experimental data was only measured at 19x11 points in a small region (Fig.16). For the elements of the correction matrix not having the corresponding experimental data, an interpolation strategy was applied. These elements can have an important influence on the identification of the machine model with defects. However, the final result of the calibration process reveals a deviation (Fig. 18 and 19) similar to that observed on the measurement results carried out on the reference plate (Fig. 8). This is a very interesting finding and allows us to confirm the performance of the proposed in-situ measurement method.

A final point concerns the used laser source, which is different from that used in industrial machines. In the current study, a red laser with a wavelength of $633 \mathrm{~nm}$ in the visible range was used. On the other hand, in a commercial LPBF machine, the used laser has a wavelength of 1070 $\mathrm{nm}$ and located in the infrared range; therefore it is not visible with the used camera. One of possible variation sources in the optical path is due to a variation in the refractive index used in [7]. For the silica glass used in the machine prototype, it is possible from the Sellmeier's formula to compute the refractive index [56, 57] for the two laser source: $n=1.4551$ for the $633 \mathrm{~nm}$ wavelength, and $n^{\prime}=1.4496$ for the $1070 \mathrm{~nm}$ wavelength. The difference of the laser spot location according to these two indexes with the model without defects is represented in Fig. 20. The results highlight the very small influence of the wavelength variation on the final result, which confirms our approach using a visible laser. 


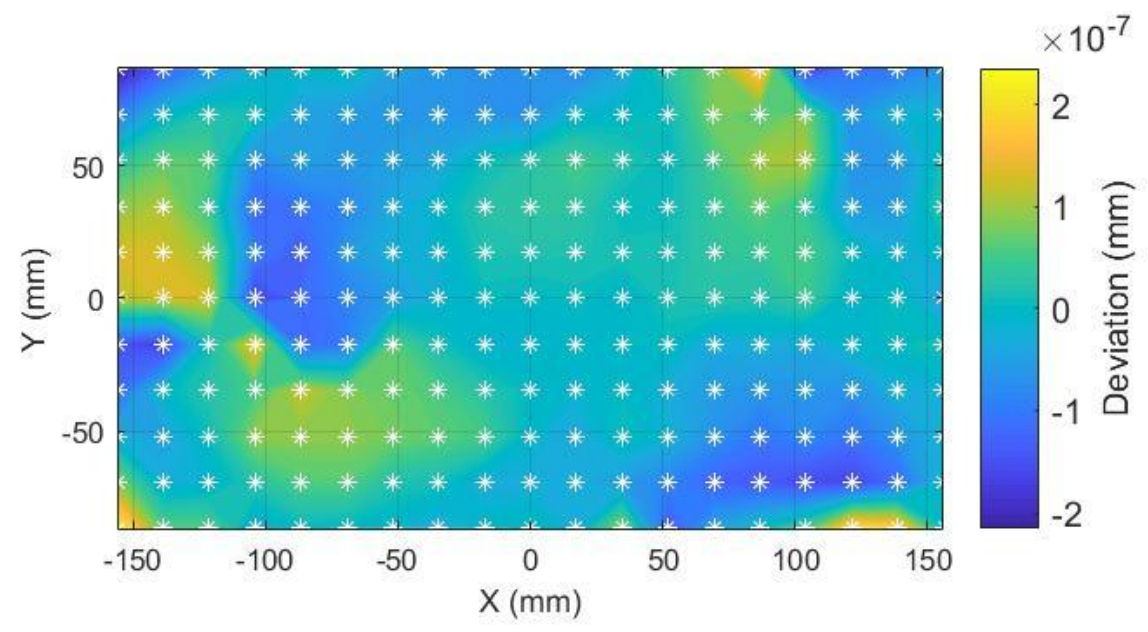

Fig. 20. Difference of the laser spot location when using two refractive indexes with the model without defects.

\section{Conclusions and future works}

In this work, we proposed an in-situ calibration approach for the laser galvanometric scanning head of LBPF machines by using a vision system. The calibration algorithm was based on the models developed in [7]. The main contribution of this work is the development of an in-situ method to rapidly measure the positions of the laser spot used for the calibration process, and avoiding the marking and measuring phases of laser marks as in previous works. The obtained results on the calibration show that the position errors between the positions generated by the identified machine with defects and those measured by our method are very small with an average error of $0.03 \mathrm{~mm}$ and the maximum position error less than $0.075 \mathrm{~mm}$. There are approximately $80 \%$ of positions with an error less than $0.04 \mathrm{~mm}$.

The calibration results to date are not yet satisfactory with the constraints required by the manufacturer due to some reasons related to the difference related to the geometry between the used machine prototype and real industrial machines. However, by comparing between the identified model with defects and the measurement, we can confirm that the developed method is acceptable for the in-situ measurement with a good accuracy level.

In future work, the calibration area in the work plane and the number of laser spot position should be extended and augmented as in [7] to obtain a sufficiently measured data and enhance the accuracy of the calibration process. Moreover, the proposed method will be implemented with an industrial LBPF machine, which has a geometric model suited with the algorithm developed in [7] to confirm the effectiveness of the proposed method in terms of time and accuracy. Moreover, compared to other calibration methods, the method we propose also allows process monitoring 
based on the geometric link between the camera mark and the production drawing mark. This aspect will also be developed in future work.

\section{References}

[1] M.-F. Chen, Y.-P. Chen, W.-T. Hsiao, S.-Y. Wu, C.-W. Hu, Z.-P. Gu, A scribing laser marking system using DSP controller, Opt. Lasers Eng. 46 (2008) 410-418. https://doi.org/10.1016/j.optlaseng.2007.11.010.

[2] W.-T. Hsiao, S.-F. Tseng, C.-K. Chung, P.-H. Chen, M.-F. Chen, Development of portable laser machining system for laser writing applications, Opt. Rev. 20 (2013) 167-172. https://doi.org/10.1007/s10043-013-0027-0.

[3] J. Ai, M. Lv, M. Jiang, J. Liu, X. Zeng, Focused laser lithographic system for efficient and cross-scale fabrication of large-area and 3D micro-patterns, Opt. Lasers Eng. 107 (2018) 335-341. https://doi.org/10.1016/j.optlaseng.2018.04.010.

[4] S. Cui, X. Zhu, W. Wang, Y. Xie, Calibration of a laser galvanometric scanning system by adapting a camera model, Appl. Opt. 48 (2009) 2632. https://doi.org/10.1364/AO.48.002632.

[5] M.F. Chen, Y.P. Chen, Compensating technique of field-distorting error for the CO2 laser galvanometric scanning drilling machines, Int. J. Mach. Tools Manuf. 47 (2007) 1114-1124. https://doi.org/10.1016/j.ijmachtools.2006.09.015.

[6] J.S. Ehrmann, Optics for vector scanning, in: Proc. SPIE 1454, Beam Deflection Scanning Technol., 1991: pp. 245-256. https://doi.org/10.1117/12.28036.

[7] K. Godineau, S. Lavernhe, C. Tournier, Calibration of galvanometric scan heads for additive manufacturing with machine assembly defects consideration, Addit. Manuf. 26 (2019) 250257. https://doi.org/10.1016/j.addma.2019.02.003.

[8] Y. Mao, L. Zeng, J. Jiang, C. Yu, Plane-constraint-based calibration method for a galvanometric laser scanner, Adv. Mech. Eng. 10 (2018) 1-11. https://doi.org/10.1177/1687814018773670.

[9] S. Yang, L. Yang, G. Zhang, T. Wang, X. Yang, Modeling and Calibration of the Galvanometric Laser Scanning Three-Dimensional Measurement System, Nanomanufacturing Metrol. 1 (2018) 180-192. https://doi.org/10.1007/s41871-018-0020-0.

[10] S. Lüdtke, B. Wagner, R. Bruder, P. Stüber, F. Ernst, A. Schweikard, T. Wissel, Calibration of Galvanometric Laser Scanners Using Statistical Learning Methods, in: H. Handels, T.M. Deserno, H.-P. Meinzer, T. Tolxdorff (Eds.), Can. J. Public Heal., Springer Berlin Heidelberg, Berlin, Heidelberg, 2015: pp. 467-472. https://doi.org/10.1007/978-3-662- 
46224-9_80.

[11] J. Tu, L. Zhang, Effective data-driven calibration for a galvanometric laser scanning system using binocular stereo vision, Sensors (Switzerland). 18 (2018). https://doi.org/10.3390/s18010197.

[12] J. Xie, S. Huang, Z. Duan, Y. Shi, S. Wen, Correction of the image distortion for laser galvanometric scanning system, Opt. Laser Technol. 37 (2005) 305-311. https://doi.org/10.1016/j.optlastec.2004.04.012.

[13] A. Manakov, H.S. Ivo, A Mathematical Model and Calibration Procedure for Galvanometric Laser Scanning Systems, Vision, Model. Vis. (2011) 207-214. https://doi.org/10.2312/pe/vmv/vmv11/207-214.

[14] P. Brosens, Scanning Accuracy of the oving-Iron Galvanometer Scanner, Opt. Eng. 15 (1976). https://doi.org/10.1117/12.7971926.

[15] F. Blais, Control Of Low Inertia Galvanometers For High Precision Laser Scanning Systems, Opt. Eng. 27 (1988). https://doi.org/10.1117/12.7976653.

[16] V.P. Bessmeltsev, N. V Goloshevsky, V. V Kasterov, Y.A. Kipriyanov, K.K. Smirnov, Method of Calibration of a Laser-Based Galvanometric Scanning System with Submicron Resolution, Optoelectron. Instrum. Data Process. 54 (2018) 390-396. https://doi.org/10.3103/S8756699018040118.

[17] J.R. Weisz, CALIBRATING LASER TRIMMING APPARATUS, 4918284, 1990.

[18] Y. Zhang, J.Y.H. Fuh, D. Ye, G.S. Hong, In-situ monitoring of laser-based PBF via off-axis vision and image processing approaches, Addit. Manuf. 25 (2019) 263-274. https://doi.org/10.1016/j.addma.2018.10.020.

[19] H.-W. Hsu, Y.-L. Lo, M.-H. Lee, Vision-based inspection system for cladding height measurement in Direct Energy Deposition (DED), Addit. Manuf. 27 (2019) 372-378. https://doi.org/10.1016/j.addma.2019.03.017.

[20] L. Dubreuil, Y. Quinsat, C. Lartigue, Calibration based on part set-up measurement for onmachine inspection using vision, Int. J. Interact. Des. Manuf. 9 (2015) 317-323. https://doi.org/10.1007/s12008-015-0290-3.

[21] G. Tapia, A. Elwany, A Review on Process Monitoring and Control in Metal-Based Additive Manufacturing, J. Manuf. Sci. Eng. 136 (2014) 060801. https://doi.org/10.1115/1.4028540.

[22] Z.Y. Chua, I.H. Ahn, S.K. Moon, Process monitoring and inspection systems in metal additive manufacturing: Status and applications, Int. J. Precis. Eng. Manuf. - Green Technol. 4 (2017) 235-245. https://doi.org/10.1007/s40684-017-0029-7.

[23] M. Grasso, B.M. Colosimo, Process defects and in situ monitoring methods in metal powder 
bed fusion: a review, Meas. Sci. Technol. 28 (2017) 044005. https://doi.org/10.1088/13616501/aa5c4f.

[24] S.K. Everton, M. Hirsch, P. Stravroulakis, R.K. Leach, A.T. Clare, Review of in-situ process monitoring and in-situ metrology for metal additive manufacturing, Mater. Des. 95 (2016) 431-445. https://doi.org/10.1016/j.matdes.2016.01.099.

[25] L. Scime, J. Beuth, Anomaly detection and classification in a laser powder bed additive manufacturing process using a trained computer vision algorithm, Addit. Manuf. 19 (2018) 114-126. https://doi.org/10.1016/j.addma.2017.11.009.

[26] L. Scime, J. Beuth, A multi-scale convolutional neural network for autonomous anomaly detection and classification in a laser powder bed fusion additive manufacturing process, Addit. Manuf. 24 (2018) 273-286. https://doi.org/10.1016/j.addma.2018.09.034.

[27] B. Zhang, J. Ziegert, A. Davies, In situ surface metrology of laser powder bed fusion processes using fringe projection, Proc. - ASPE/Euspen 2016 Summer Top. Meet. Dimens. Accuracy Surf. Finish Addit. Manuf. (2016).

[28] B. Zhang, W.S.L. Ii, J. Ziegert, A. Davies, In Situ Monitoring of Laser Powder Bed Fusion Additive Manufacturing Using Digital Fringe Projection Technique, Proc. ASPE 2015 Spring Top. Meet. (2015) 47-52.

[29] B. Zhang, J. Ziegert, F. Farahi, A. Davies, In situ surface topography of laser powder bed fusion using fringe projection, Addit. Manuf. 12 (2016) 100-107. https://doi.org/10.1016/j.addma.2016.08.001.

[30] Y. Zhang, G.S. Hong, D. Ye, K. Zhu, J.Y.H. Fuh, Extraction and evaluation of melt pool, plume and spatter information for powder-bed fusion AM process monitoring, Mater. Des. 156 (2018) 458-469. https://doi.org/10.1016/j.matdes.2018.07.002.

[31] M.A. Ortega Delgado, A.F. Lasagni, Reducing field distortion for galvanometer scanning system using a vision system, Opt. Lasers Eng. 86 (2016) 106-114. https://doi.org/10.1016/j.optlaseng.2016.05.016.

[32] J.-P. Kruth, S. Dadbakhsh, B. Vrancken, K. Kempen, J. Vleugels, J. Van Humbeeck, Additive Manufacturing of Metals via Selective Laser Melting: Process Aspects and Material Developments, in: Addit. Manuf., CRC Press, 2015: pp. 69-99. https://doi.org/10.1201/b19360-4.

[33] Y. Quinsat, L. Dubreuil, C. Lartigue, A novel approach for in-situ detection of machining defects, Int. J. Adv. Manuf. Technol. 90 (2017) 1625-1638. https://doi.org/10.1007/s00170016-9478-3.

[34] J. Heikkila, O. Silven, A four-step camera calibration procedure with implicit image 
correction, in: Proc. IEEE Comput. Soc. Conf. Comput. Vis. Pattern Recognit., IEEE Comput. Soc, n.d.: pp. 1106-1112. https://doi.org/10.1109/CVPR.1997.609468.

[35] Z. Zhang, A flexible new technique for camera calibration, IEEE Trans. Pattern Anal. Mach. Intell. 22 (2000) 1330-1334. https://doi.org/10.1109/34.888718.

[36] R. Usamentiaga, D.F. Garcia, C. Ibarra-Castanedo, X. Maldague, Highly accurate geometric calibration for infrared cameras using inexpensive calibration targets, Measurement. 112 (2017) 105-116. https://doi.org/10.1016/j.measurement.2017.08.027.

[37] Y. Wang, B. Cai, K. Wang, X. Chen, Out-of-focus color camera calibration with one normalsized color-coded pattern, Opt. Lasers Eng. 98 (2017) 17-22. https://doi.org/10.1016/j.optlaseng.2017.05.017.

[38] Q. Sun, Y. Hou, Q. Tan, A new method of camera calibration based on the segmentation model, Optik (Stuttg). 124 (2013) 6991-6995. https://doi.org/10.1016/j.ijleo.2013.05.179.

[39] L. Yu, Y. Han, H. Nie, Q. Ou, B. Xiong, A calibration method based on virtual large planar target for cameras with large FOV, Opt. Lasers Eng. 101 (2018) 67-77. https://doi.org/10.1016/j.optlaseng.2017.10.003.

[40] R. Hartley, A. Zisserman, Multiple View Geometry in Computer Vision, Cambridge University Press, Cambridge, 2004. https://doi.org/10.1017/CBO9780511811685.

[41] A. Vyas, M.B. Roopashree, B.R. Prasad, Centroid Detection by Gaussian Pattern Matching in Adaptive Optics, Int. J. Comput. Appl. 1 (2010) 32-37. https://doi.org/10.5120/483-793.

[42] J. Zhang, Research on the measurement accuracy of different laser spot center location, in: A. Tian (Ed.), Third Int. Conf. Photonics Opt. Eng., SPIE, 2019: p. 38. https://doi.org/10.1117/12.2521709.

[43] C. Tang, C. Hou, Z. Song, Defocus map estimation from a single image via spectrum contrast, Opt. Lett. 38 (2013) 1706. https://doi.org/10.1364/ol.38.001706.

[44] P. Zhou, X. Wang, Q. Huang, C. Ma, Laser Spot Center Detection Based on Improved Circled Fitting Algorithm, Proc. 2018 2nd IEEE Adv. Inf. Manag. Commun. Electron. Autom. Control Conf. IMCEC 2018. (2018) 316-319. https://doi.org/10.1109/IMCEC.2018.8469554.

[45] S.M. Thomas, Y.T. Chan, A simple approach for the estimation of circular arc center and its radius, Comput. Vision, Graph. Image Process. 45 (1989) 362-370. https://doi.org/10.1016/0734-189X(89)90088-1.

[46] F. Qian, X.-P. Zhang, Precision improved spot centroid-locating algorithm based on profile regularization, in: 4th Int. Conf. Electr. Electron. Eng. Comput. Sci. (ICEEECS 2016), Atlantis Press, Paris, France, 2016: pp. 849-856. https://doi.org/10.2991/iceeecs- 
16.2016.165.

[47] H. Dong, L. Wang, Non-iterative spot center location algorithm based on Gaussian for fisheye imaging laser warning system, Optik (Stuttg). 123 (2012) 2148-2153. https://doi.org/10.1016/j.ijleo.2011.10.012.

[48] B. Hou, Charge-coupled devices combined with centroid algorithm for laser beam deviation measurements compared to a position-sensitive device, Opt. Eng. 50 (2011) 033603. https://doi.org/10.1117/1.3554379.

[49] Z. Liu, B.K. Huang, Y.N. Shang, G.J. Zhang, A high precision laser spot center location method based on multi-scale image analysis, Adv. Intell. Soft Comput. 133 AISC (2012) 1019-1025. https://doi.org/10.1007/978-3-642-27552-4_133.

[50] A. Vázquez-Otero, D. Khikhlukha, J. Solano-Altamirano, R. Dormido, N. Duro, Laser Spot Detection Based on Reaction Diffusion, Sensors. 16 (2016) 315. https://doi.org/10.3390/s16030315.

[51] R. Singh, J.M. Hattuniemi, A.J. Mäkynen, Analysis of accuracy of laser spot centroid estimation, Adv. Laser Technol. 2007. 7022 (2008) 702216. https://doi.org/10.1117/12.804104.

[52] S. Thomas, Optimized centroid computing in a Shack-Hartmann sensor, in: Adv. Adapt. Opt., 2004: p. 1238. https://doi.org/10.1117/12.550055.

[53] A. Stratan, A. Zorila, L. Rusen, G. Nemes, Measuring effective area of spots from pulsed laser beams, Opt. Eng. 53 (2014) 122513. https://doi.org/10.1117/1.OE.53.12.122513.

[54] F.M. Dickey, T.E. Lizotte, Laser Beam Shaping Applications, CRC Press, 2017.

[55] N.C. du Preez, A. Forbes, L.R. Botha, High power infrared super-Gaussian beams: generation, propagation, and application, in: XVII Int. Symp. Gas Flow, Chem. Lasers, HighPower Lasers, 2008: p. 71311E. https://doi.org/10.1117/12.820058.

[56] I. H. Malitson. Interspecimen comparison of the refractive index of fused silica, J. Opt. Soc. Am 1965; 55: 1205-1208.

[57] C. Z. Tan. Determination of refractive index of silica glass for infrared wavelengths by IR spectroscopy, J. Non-Cryst. Solids 1998;223: 158-163. 